\title{
Optimizing Hierarchical Power Distribution of Multiple Local Energy Network Systems in Grid-Connected Mode
}

\author{
Zhang Fuxing1*, Zhang Tao ${ }^{1,2}$, Wang Rui ${ }^{1}$ \\ ${ }^{1}$ College of Information Systems and Management, National University of Defense Technology, \\ Changsha, P.R. China, 410073 \\ ${ }^{2}$ State Key Laboratory of High-Performance Computing, National University of Defense Technology, \\ Changsha, P.R. China, 410073
}

Received: 7 February 2017

Accepted: 12 March 2017

\begin{abstract}
This paper focuses on the hierarchical power distribution optimization of multiple local energy network (LEN) systems that are formed in three levels and can be operated in six typical modes. The decentralized optimal model for each LEN (the first level) and LENs (the second level) as well as the concentrated optimal model for the top level of the system are built, respectively. For each LEN, all the basic unities such as power generated by wind turbines and photovoltaic, and their upper nodes are considered. For LENs, the aggregated results (e.g., supply-demand requirements) from each LEN are dispatched. Furthermore, in the concentrated optimal control model (the third level), the ultimate supply-demand requirements of networked LENs together with other resources such as electric vehicles are considered. Due to the large number of control resources, the whole system is formulated as a large-scale global optimization (LSGO) problem. The self-adaptive differential evolution with neighborhood search method (SaNSDE) modified with the Lagrange multiplier method is used to solve the problem. The algorithm is firstly examined on 10 constrained benchmark functions, then it is applied to our problem. Experimental results show that the proposed model and algorithm are effective and efficient.
\end{abstract}

Keywords: local energy network (LEN), energy internet (EI), decentralized renewable energy sources (DRES), adaptive neighborhood search technique, electric vehicles (EVs)

\section{Introduction}

With increasing environmental pollution and the reduction of fossil energy storage, decentralized renewable energy sources (DRES) have been considered to be effective alternatives for future energy requirements

*e-mail: zfx@nudt.edu.cn
[1-2]. For adapting to the characteristics of DRES (e.g., geographical dispersion), local energy network systems (LENs) are employed to strengthen the collaborative relationship between DRES and the smart grid. LENs are composed of many small-scale but self-governing LENs whose basic members include renewable energy production units (e.g., wind turbines and photovoltaic generators), controllable storage units, and intelligent consumption units [3-4]. Different LENs are coupled 
into networked energy transmission and distribution systems, which are called the energy internet (EI). LENs can satisfy the requirements of future power distribution and operation both in grid-connected and autonomous modes where power and information flows are bilaterally exchanged and the power scheduling changes are more flexible, economic, and environmental [5-7]. Especially in the grid-connected mode, LENs can not only take advantage of the powerful transmission and distribution capability of the existing power grid, but also mitigate the uncertainty of DRES by constructing more convenient and reliable distribution networks as well as the schedulable resources on the user side, such as demand response (DR).

Many studies have discussed the optimization of a single LEN for utilizing DRES along with a distributed battery energy storage system (BESS), which were considered as promising techniques and cost-effective ways to strengthen environmental protection and adapt rapid economic growth, especially when the penetration ratio of DRES and BESS are high. [8] has put forward an advanced cooperative operation strategy for BESS in single LEN, which can not only balance the requirements of supply-demand, but also make full use of BESS to reduce the power loss of transmission lines. However, its adjustment ability of arranging internal schedulable units to keep supply and demand balance was finite, which needed to be improved by considering the load consumption prediction and other dispatch methods, such as DR. The current literatures includes several ways for a single LEN to achieve self-balance:

1. Continually changing charging/discharging process of BESS.

2. Reducing the requirements of partial loads, called interrupted loads (ILs).

3. Augmenting the capacity and/or quantity of electric generators.

4. Increasing the exchange frequency with its connected medium voltage power grid.

However, all of above four strategies not only increase operational costs as well as power dispatch difficulty, but they decrease the internal reliability of a single LEN. Moreover, due to lacking of wired/wireless communication networks, a single LEN cannot receive global information from its neighbor and/or upper connected power grid and can hardly transmit its message to others, which significantly influences its interconnection performance and is not suitable for the plug-and-play feature of utilizing DRES. So an efficient power distribution system should be hierarchical and intelligent, which not only couples the existing smart grid and large-scale single LEN into a controllable energy distribution network, but also contributes to bidirectional exchange for intricate information as well as power flows.

Recent research has also noticed the process of information communication and power distribution among different LENs, multiple LENs, and the utility grid, which considers the access of high proportional DRES into a regional power distribution system and relevant scheduling strategies, such as operating in islanded mode [9-10], grid-connected mode[11], or both [12]. Due to sufficient information sharing and rapid power exchange, multiple LENs have a stronger ability to keep internal balance than that of a single LEN, which also has a unique selfhealing ability in some emergency situations. Meanwhile, multiple LENs can obtain other more economic values and social benefits in grid-connected mode, such as reducing unnecessary electric consumption, postponing the investment of the existing power grid, increasing utilization efficiency of the network, and strengthening environmental protection. So the grid-connected multiple LENs have many advantages for efficiently utilizing largescale DRES, implementing different scheduling plans, and adapting future various requirements about saving energy and reducing emissions.

Currently, how to design an advanced molding and solving method for optimizing the power distribution among networked multiple LENs is all-important and widely discussed. With this motivation, efforts on architecture design, molding, and solving methods of networked multiple LENs have been drawn much more attention:

1. For the architecture design issue, [13] has reviewed the difference between and integrated energy system (IES) and EI: IES focused on the interaction process between natural gas infrastructures and power electricity systems, while EI paid more attention on the function used for energy distribution, such as cyber-physical systems, energy router, and internet-based technology. In [14], the hierarchical control framework of the energy management system (EMS) has been proposed to meet the new requirements of networked multiple LENs, which integrate a number of advanced techniques such as progressive metering technology, highly reliable communication technology, load consumption prediction technology, big data processing technology, and energy scheduling technology based on model predictive control. The authors in [15] presented a new coordinative operation architecture of multiple LENs, which emphasize the interaction of generation/consumption units, power distribution grid, and the energy storage system. The study in [16] gave a decentralized EMS of interconnected LENs, both in grid-connected and islanded modes.

2. For the molding method issue, the multi-agent system (MAS)-based control scheme, optimization strategy, and corresponding solving method were discussed in [17-18]. The research in [19] has analyzed the molding method based on the available transfer capability (ATC) of a large networked power grid, in which a decomposition method to calculate ATC was proposed. Recently, [20] presented a novel molding method of networked LENs by online energy optimization technique and robust decentralized operation strategy. [21] introduced a new model of interconnected multi-energy-grids and proposed an advanced optimization strategy to make full use of DRES. In [22], a cooperative smart grid model was 
built, which integrated the networked LENs as well as the utility grid and regarded them as a system of systems.

3. For the solving method issue, [23] proposed a decentralized optimization method to quickly eliminate the influence of sudden disturbances from DRES and/or loads for smart LENs with grid-connected, island, and synchronous modes. [24] puts forward a coordination operation technique by considering the distribution network loss and the load fluctuation. Then the particle swarm optimization method, together with Monte Carlo simulation, were considered as solutions to the problem.

From the above-mentioned papers we know that the modeling and optimizing methods of networked multiple LENs are urgent and it is important to address the high uncertainty of DRES and balance supply-demand requirements. However, the modeling and optimizing methods for each level of hierarchically networked LENs have not been discussed simultaneously.

In this study, a novel hierarchical structure of networked multiple LENs in grid-connected mode is proposed and is shown in Fig. 1. This includes three levels of network: local energy network (LEN), neighborhood area network (LENs), and wide area network. By considering the bidirectional exchange of power and/or information flows, a novel modeling methodology - including two decentralized models and one concentrated model - has been proposed to describe the interactive process among the nodes of network for each level. To obtain the optimal control of the whole system, the problem is formulated as a large-scale global optimization (LSGO) problem. An effective algorithm, the self-adaptive differential evolution with neighborhood search (SaNSDE) is selected to solve the problem. The constraints in the problem are handled by the Lagrange multiplier method. The algorithm is demonstrated as an effective method for our problem.

The remainder of this paper is organized as follows: Material and Methods introduces the physical and communicational framework of networked multiple LENs in the grid-connected mode, then the general formulation of the optimization problem and our proposed optimization process as well as methods are specified and the numerical results are shown and discussed in Results and Discussion. Conclusions concludes this paper and presents our future research directions.

\section{Material and Methods}

Framework of Networked Multiple LENs

\section{Hierarchical Framework}

The hierarchical framework of networked multiple LENs is shown in Fig. 1, which is constructed by three level networks: local area network (LEN), neighborhood area network (LENs), and wide area network. The blue lines or arrows denote the information flows and are networked through EMS, by which each component can transmit or receive messages. The red lines or arrows stand for the

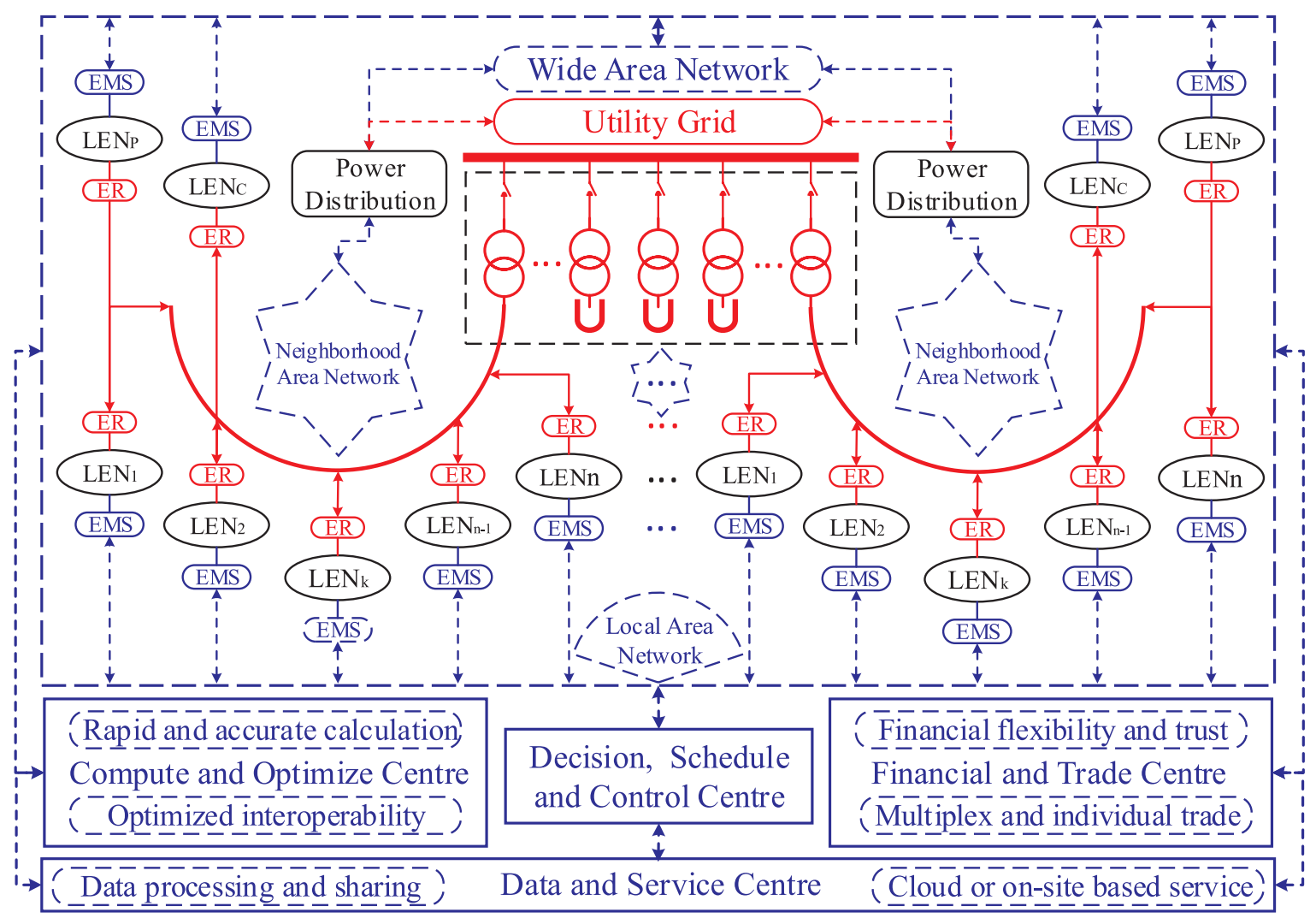

Fig 1. The hierarchical framework of networked multiple LENs in grid-connected mode. 
power flows and implement corresponding operational commands, in which different parts are connected by an energy router (ER).

Each LEN is composed of a number of interconnected $\operatorname{LEN}_{i}(i=1,2,3, \ldots, k, \ldots m)$, where the configuration amount $m$ is decided by regional production and consumption abilities. For example, if the management scope of LENs is large and it comprises a mass of loads, the configuration parameter $m$ will be huge; otherwise only a few LENs with a small quantity of production units can satisfy the requirements of all loads. $\mathrm{LEN}_{k}$ means the $k$ th subcomponent of LENs and contains several controllable/ uncontrollable elements, such as a natural gas generator (NGG), a wind-driven generator (WDG), a photovoltaic generator (PVG), BESS, electric vehicles (EVs), and intelligent loads. The local central controller (LCC) is the regional governor of each LEN and tries its best to maintain internal supply-demand balance by adjusting the output amount of production units, changing charge/ discharge rate of BESS, and controlling ILs as well as the exchange amount with external nodes, such as neighbor LEN and its connected upper node of LENs.

Distribution network operator (DNO) is employed to coordinate the input/output relationships among different LENs and their connected upper nodes of networked multiple LENs. The global central controller (GCC) is the highest coordinator and its main function is to balance the ultimate supply-demand requirements with minimum operational cost for the whole networked multiple LENs. LCC, DNO, and GCC are located in the following three levels and their interactive information is exchanged by wired/wireless communication networks, by which the networked multiple LENs can reach regional autonomy, wide-area synergy, and a global optimal state.

For better understanding the framework of networked multiple LENs in grid-connected mode, all of its structures and corresponding functions are specified as follows:

1. Local area network (LEN). For each LEN, there exists an integrated generation-grid-load-storage subcomponent. With advanced sensor technology, efficient calculation capacity, and cloud- or on-sitebased internet, exchanged information from different parts of each LEN can be gathered, collected, classified, transferred, computed, and stored. Then parts of them are changed into executable instructions by LCC to guide the course of production, transmission, distribution, and consumption. If there are sufficient/ insufficient power requirements after internal distribution, LCC will interact with other external nodes, by which LEN not only obtains abundant profit by selling its sufficient power energy or curtailing unnecessary loads, but also maintains internal balance by buying equivalent insufficient power from its neighbor LEN and/or connected upper node of LENs.

2. Neighborhood area network (LENs). For each LEN, DNO can receive the sufficient/insufficient information from its controllable LEN, in which all the LENs are strongly connected and can support each other to keep the supply-demand balance. Due to the uncertainty of
DRES and load requirements, both the production and consumption quantities of each LEN keep changing every time, which means its internal balance cannot always be met by itself. In order to keep internal balance, each LCC will exchange electric power with its neighbor LCC, which is controlled by the same DNO. For example, if the total energy requirements of a certain LEN are sufficient at simulation interval $t$, its LCC will sell the redundant part and obtain corresponding financial revenue. Otherwise, LCC will purchase the same amount of adequate power and afford equivalent cost. The distance of two exchanged LENs and the real-time price (RTP) are considered the main factors to regulate the transaction sequence among different LCCs in the same LENs.

3. Wide area network. For a cluster of or all LENs, GCC can receive their sufficient/insufficient information and balance corresponding supply-demand requirements. For example, if there is imbalance after exchanging power between sufficient and insufficient LENs, GCC will coordinate relevant LENs together with other schedulable nodes, such as traditional thermal power plants (TPP), to keep their ultimate balance. For each LEN or LENs, we assume that the buying price from its connected upper node is higher than that from its neighbor LEN or LENs, while the selling price to its connected upper node is lower than that to its neighbor LEN or LENs, which can encourage all the LEN or LENs to utilize local energy, especially for DRES. We also assume that GCC has enough ability to keep its internal balance at any time and hardly exchange power with other external nodes.

\section{Typical Operation Scene}

By summarizing the above structure of networked multiple LENs in grid-connected mode, we extract its universal structure of different level networks and give six typical operational scenes, shown as Fig. 2.

The extracted structure contains three level nodes. Among them, the sub-node layer is composed of largescale small prosumers and then their supply-demand requirements are polymerized to the lower production/ consumption node layer. If imbalance circumstance exists in any lower node, it will first exchange power with its neighbor lower node. If imbalance still exists, the remainder of the lower node will be satisfied by its connected upper node. We assume that the connected upper node has enough ability to satisfy all the requirements from the lower production/consumption node layer. According to the diversity of power transmission and distribution among the lower nodes and their connected upper node, six different scenes are expatiated as follows:

1. Self-balance state without power exchange from neighbor or connected upper node (Fig. 2a). For this state, there are two typical circumstances: on one hand, the polymerized supply requirements from sub-nodes just equal to their demand requirements, which is an ideal situation without any artificial interference. On 

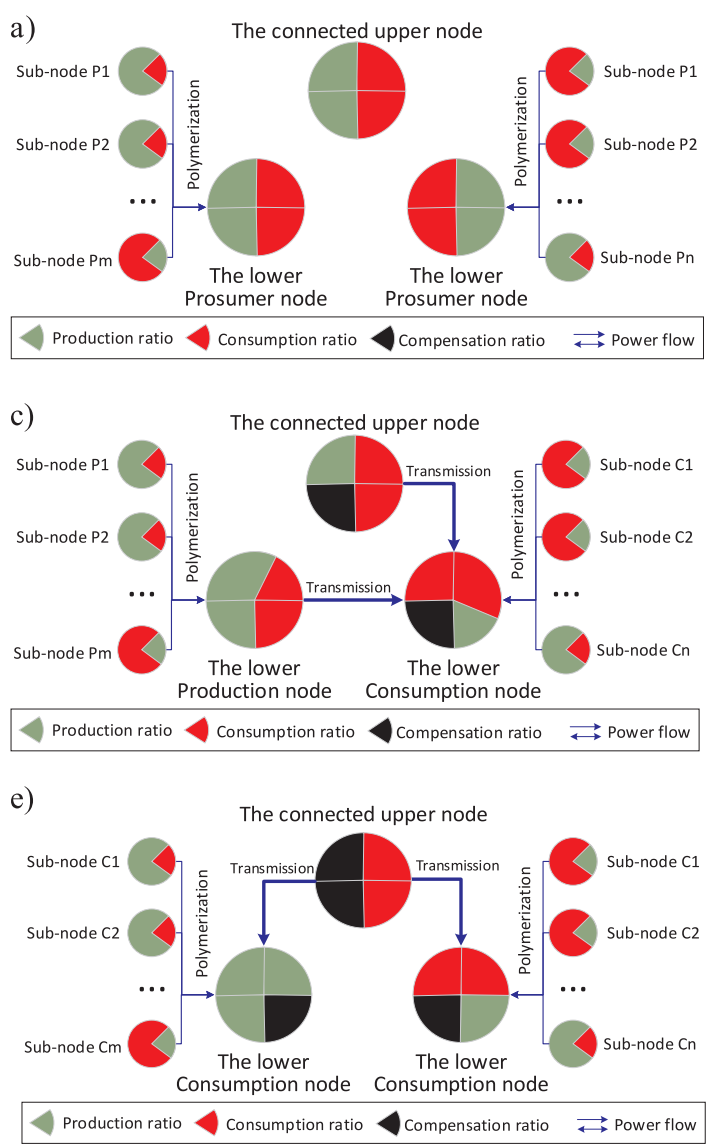
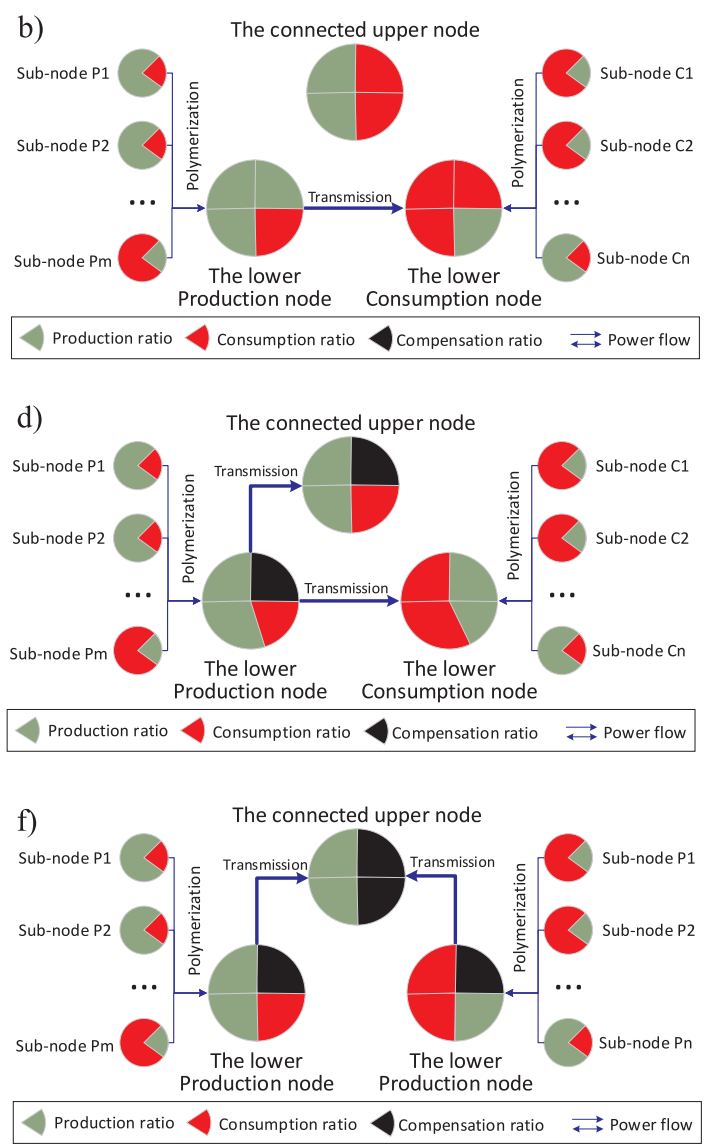

Fig. 2. Typical operational scenes of extracted structure for networked multiple LENs in grid-connected mode.

the other hand, the polymerized supply and demand requirements of each lower node are not matched, but the LCC of sub-nodes can adaptively adjust its internal controllable units (e.g., NGG, BESS, and ILs) to meet the balance, which is arbitrary and not beneficial to making full use of DRES.

2. Balance state with power exchange among lower production and consumption nodes, shown as Fig. $2 \mathrm{~b}$. For this state, the polymerized requirements of subnodes do not reach the supply-demand balance but the difference is small, which exceeds the adjustment ability of a single lower node but does not need to exchange power with its connected upper node. Then the lower nodes with sufficient power energy transmit their surplus to those insufficient lower nodes. The transmission line losses and RTP among different lower nodes are considered.

3. Balance state with internal power exchange and buying/selling power from/to the connected upper node (Figs. 2c-d). For those two states, sub-nodes first polymerize their requirements to their lower production/consumption nodes. Then the power exchange among lower nodes is complete, but their energy supply and demand requirements are still imbalanced, which requires the connected upper node to output or input equivalent power energy.

4. Balance state with buying/selling power from/to the connected upper node (Figs 2e-f). For the former state, the polymerized energy from sub-nodes are insufficient to balance their requirements, which regard all the lower nodes as consumers and their connected upper node needs to output a lot of energy to them. Conversely, the later state regards all the lower nodes as producers and their redundant power energy are output to the connected upper node.

\section{Mathematical Formulation}

\section{Optimization Model of LEN}

LEN is the minimum optimization unit of the networked multiple LENs in grid-connected mode, which is regarded as a prosumer and contains large-scale distributed generators, consumers, and BESS. Its objective function and corresponding constraints are represented as follows.

1) Objective function

The objective function of LEN is to minimize the cost and maximize the revenue within any simulation interval, whose fundamental expression is shown as follows:

$$
\begin{gathered}
\min \operatorname{Cost}_{L E N}=\sum_{t=1}^{T}\left(C_{N G G}(t)+C_{W D G}(t)+C_{P V G}(t)+C_{C L s}(t)+\right. \\
\left.+C_{I L s}(t)+C_{B E S S}(t)+C_{\text {NeiLEN }}(t)+C_{U p N o d e}(t)\right)
\end{gathered}
$$


...where $T$ stands for the total simulation interval and $\operatorname{Cost}_{L E N}$ denotes the objective value. On the right side of equation (1), the first three items mean the cost of production units. The fourth and fifth items present the cost of consumption units. The sixth item shows the charge/discharge cost of BESS. The last two items give the exchange cost between LEN and its external nodes. Note that the positive value means the cost and the negative one presents the revenue in the objective function above.

2) Energy balance constraints

The output electricity power must be consumed in a timely way and cannot be stored for a long time, which makes it a big challenge to balance energy production and consumption every time [25]. For each LEN, the energy balance constraint is represented as equation (2), in which electrical power is generated by the controllable/ uncontrollable distributed generators, the discharge process of BESS, and the input energy from external power grids, while the consumption parts include the usage of intelligent loads, the charge process of BESS, and the output energy to other external grids, such as its neighbor LEN or connected upper node of LENs.

For keeping the internal balance among different components of LEN, controllable generators, BESS, and intelligent loads are adjustable by corresponding LCC. The energy balance equation for LEN can be represented as follows:

$$
\begin{gathered}
\sum_{\text {Gi }=1}^{G n} P_{G i}(t)+\sum_{\text {DisChi }=1}^{\text {DisChn }} P_{\text {DisChi }}(t)+\sum_{\text {Buyi }=1}^{\text {Buyn }} P_{\text {Buyi }}(t)=\sum_{\text {Li } i=1}^{L n} P_{L i}(t)+ \\
+\sum_{\text {Chi } 1}^{\text {Chn }} P_{\text {Chi }}(t)+\sum_{\text {Selli } i=1}^{\text {Selln }} P_{\text {Selli }}(t)
\end{gathered}
$$

... where $P_{G i}(t)$ and $P_{L i}(t)$ stand for the output power of the $G i$ th generation and input power of the $L i$ th consumption unit, respectively. $P_{D i s C h i}(t)$ and $P_{C h i}(t)$ represent the discharging/charging power of the DisChi th and Chi th BESS. Note that the BESS cannot be in the charging and discharging state simultaneously. $P_{\text {Buyi }}(t)$ and $P_{\text {Selli }}(t)$ mean the exchanged power with its neighbor LEN and/or connected upper node of LENs during simulation interval $t$.

3) Distributed generator constraints

Each LEN includes two categories of distributed generators: controllable and uncontrollable units. The former includes NGG, while the later contains WDG and PVG.

For NGG, its operating cost of each unit power is changeable with the fluctuation of DRES and load requirements. Its adjustable scope is limited between upper $P_{N G G i}^{\max }(t)$ and lower $P_{N G G i}^{\min }(t)$ bounds. The cost of NGG $C_{N G G}(t)$ during simulation interval $t$ is a quadratic equation and the decision variable $P_{N G G i}(t)$ presents its output power, shown as follows:

$$
C_{N G G}(t)=\sum_{N G G i=1}^{N G G n}\left(\alpha_{N G G i} * P_{N G G i}(t)^{2}+\beta_{N G G i} * P_{N G G i}(t)+\gamma_{N G G i}\right)
$$

$$
P_{N G G i}^{\min }(t) \leq P_{N G G i}(t) \leq P_{N G G i}^{\max }(t)
$$

...where $C_{N G G}(t)$ presents the cost of all NGGs during simulation interval $t . \alpha_{N G G i}, \beta_{N G G i}$, and $\gamma_{N G G i}$ present the quadratic, monomial, and constant coefficients. $P_{N G G}(t)$ indicates the output power of the $N G G i$ th NGG. We assume that $C_{N G G}(t)$ includes the cost of consumptive natural gas, initial construction cost, wages of workers, and operational cost.

The costs of both WDG and PVG contain two parts. The former includes initial construction cost and wages of workers, while the later part means the operational cost, which is shown as following equations:

$$
\begin{array}{r}
C_{W D G}(t)=\sum_{W D G i=1}^{W D G n}\left(\frac{\operatorname{Cost}_{W D G i}^{i n i} / S L_{W D G i}+\operatorname{Cost}_{W D G i}^{w a g}}{365 * \operatorname{Sim} T}+\operatorname{Cost}_{W D G i}^{o p e r} *\right. \\
\left.* P_{W D G i}(t)\right)
\end{array}
$$

$$
\begin{gathered}
C_{P V G}(t)=\sum_{P V G i=1}^{P V G n}\left(\frac{\operatorname{Cost}_{P V G i}^{i n i} / S L_{P V G i}+\operatorname{Cost}_{P V G i}^{w a g}}{365 * \operatorname{Sim} T}+\operatorname{Cost}_{P V G i}^{\text {oper }} *\right. \\
\left.* P_{P V G i}(t)\right) \\
P_{W D G i}^{\min }(t) \leq P_{W D G i}(t) \leq P_{W D G i}^{\max }(t) \\
P_{P V G i}^{\min }(t) \leq P_{P V G i}(t) \leq P_{P V G i}^{\max }(t)
\end{gathered}
$$

...where $C_{W D G}(t)$ and $C_{P V G}(t)$ present all the cost of WDGs and PVGs, and $P_{W D G i}(t)$ and $P_{P V G i}(t)$ stand for the output power of the WDGi th and $P V G i$ th distributed generators during simulation interval $t$, respectively. Cost $t_{W D G i}^{i n i}$, $\operatorname{Cost}_{W D G i}^{\text {wag }}, \operatorname{Cost}_{W D G i}^{\text {oper }}, \operatorname{Cost}_{P V G i}^{i n i}, \operatorname{Cost}_{P V G i}^{\text {wag }}$ and $\operatorname{Cost}_{P V G i}^{\text {oper }}$

show the initial construction cost, wages of workers, and operational cost of the WDGi th and PVGi th distributed generators. $S L_{W D G i}$ and $S L_{P V G i}$ denote the service life. Sim T presents all the simulation intervals. $P_{W D G i}^{\min }(t), P_{P V G i}^{\min }(t)$, $P_{W D G i}^{\max }(t)$, and $P_{P V G i}^{\max }(t)$ give the lower and upper bounds of decision variable $P_{W D G i}(t)$ and $P_{P V G G i}(t)$.

Considering the transformation efficiency and transmission loss, the practical output of distributed generators are less than the above theoretical values, which are calculated by the following equations:

$$
P_{N G G}^{\text {practical }}(t)=\left\{\begin{array}{cc}
\eta_{N G G}^{H} * P_{N G G}(t) & \text { if } P_{N G G}^{\max }(t) * 2 / 3 \leq P_{N G G}(t) \leq P_{N G G}^{\max }(t) \\
\eta_{N G G}^{L} * P_{N G G}(t) & \text { if } P_{N G G}^{\min }(t) \leq P_{N G G}(t) \leq P_{N G G}^{\max }(t) / 3 \\
\eta_{N G G}^{M} * P_{N G G}(t) & \text { Otherwise }
\end{array}\right.
$$




$$
P_{W D G}^{\text {practical }}(t)=\left\{\begin{array}{lc}
\eta_{W D G}^{H} * P_{W D G}(t) & \text { if } P_{W D G}^{\max }(t) * 2 / 3 \leq P_{W D G}(t) \leq P_{W W G}^{\max }(t) \\
\eta_{W D G}^{L} * P_{W D G}(t) & \text { if } P_{W D G}^{\min }(t) \leq P_{W D G}(t) \leq P_{W D G}^{\max }(t) / 3 \\
\eta_{W D G}^{H} * P_{W D G}(t) & \text { Otherwise }
\end{array}\right.
$$

$$
P_{P V G}^{\text {practical }}(t)=\left\{\begin{array}{lc}
\eta_{P V G}^{H} * P_{P V G}(t) & \text { if } P_{P V G}^{\max }(t) * 2 / 3 \leq P_{P V G}(t) \leq P_{P P G}^{\max }(t) \\
\eta_{P V G}^{L} * P_{P V G}(t) & \text { if } P_{P V G}^{\min }(t) \leq P_{P V G}(t) \leq P_{P V G}^{\max }(t) / 3 \\
\eta_{P V G}^{H} * P_{P V G}(t) & \text { Otherwise }
\end{array}\right.
$$

...where $\eta_{*}^{H}, \eta_{*}{ }^{M}$, and $\eta_{*}{ }^{L}$, mean that the transformation efficiency of NGG, WDG, and PVG are in the high, middle, and low states, which also indicate that their transmission loss are in the low, middle, and high states. Piecewise functions (9)-(11) classify the practical production capacity into three parts and show different production efficiencies, where the higher production amount corresponds to higher production efficiency and lower transmission loss.

4) Intelligent load constraints

It is assumed that each LEN contains two kinds of intelligent loads: crucial loads (CLs) and ILs. CLs are given higher reliability of power supply with a higher rate of RTP, which are not allowed to be interrupted or transformed. ILs can be partially curtailed when demand exceeds supply. But the interrupted part can get corresponding compensatory payment, which is higher than RTP but lower than the exchange price between LEN and its connected upper node of LENs.

$$
\begin{gathered}
C_{C L \mathrm{~s}}(t)=\sum_{C L s i=1}^{C L s n}\left(-P_{C L s i}(t) * \text { PRate }_{C L s i}(t)^{*} \text { price }_{R T P}(t)\right) \\
C_{I L \mathrm{~s}}(t)=\sum_{I L s i=1}^{I L s n}\left(-P_{I L s i}(t) *\left(1-\text { LRate }_{I L s i}(t)\right) * \text { price }_{R T P}(t)+\right. \\
\left.+P_{I L s i}(t)^{*} \text { LRate }_{I L s i}(t) * \text { PRate }_{I L s i}(t) * \text { price }_{R T P}(t)\right)
\end{gathered}
$$

...where $C_{C L s}(t), C_{I L s}(t), P_{C L s i}(t)$, and $P_{I L s i}(t)$ represent the cost and electric power of CLs and ILs, respectively. PRate $_{C L S i}(t)$ and PRate $_{I L S i}(t)$ stand for the compensation rate of CLs and ILs, which are slightly higher than RTP. LRate $_{I L s i}(t)$ denotes the interrupted rate of ILs and price $_{\text {RTP }}(t)$ indicates RTP.

5) BESS constraints

BESS in each LEN is scheduled by LCC to alleviate the fluctuation between the production and consumption side. BESS not only charges power when the production amount is sufficient, but it also discharges power to loads when the supply falls short of demand. The cost of BESS contains both constant and variable parts, in which the former is expressed by limited charge/discharge times and its initial construction cost, while the later includes the charge/discharge efficiency and self-discharge rate.

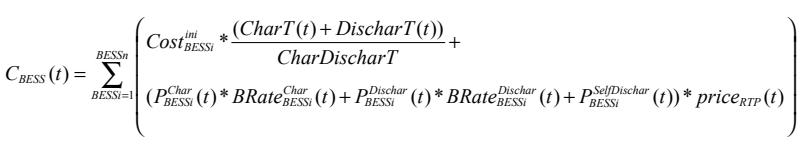

$$
\begin{gathered}
P_{B E S S i}^{\text {Char min }}(t) \leq P_{B E S S i}^{\text {Char }}(t) \leq P_{B E S S i}^{\text {Charm } \max }(t) \\
P_{B E S S i}^{\text {Dischar min }}(t) \leq P_{B E S S i}^{\text {Dischar }}(t) \leq P_{B E S S i}^{\text {Dischar max }}(t)
\end{gathered}
$$

$\operatorname{Cap}_{B E S S i}(t+1)=\operatorname{Cap}_{B E S S i}(t)+P_{B E S S i}^{\text {Char }}(t)-P_{B E S S i}^{\text {Dischar }}(t)-P_{B E S S i}^{\text {Selfischar }}(t)$

$$
\operatorname{Cap}_{B E S S i}^{\min }(t) \leq \operatorname{Cap}_{B E S S i}(t) \leq \operatorname{Cap}_{B E S S i}^{\max }(t)
$$

...where $C_{B E S S}(t)$ and $\operatorname{Cost} t_{B E S S i}^{i n i}$ mean the total cost and the initial construction cost of BESS. CharT $(t)$ and $\operatorname{DischarT}(t)$ indicate the charge and discharge times during simulation interval $t$, while CharDischarT denotes the total available charge and discharge times during its service lifetime. $P_{B E S S i}^{\text {Char }}(t), P_{B E S S i}^{\text {Dischar }}(t)$, and $P_{B E S S i}^{\text {Selfischar }}(t)$ present the charge power, discharge power, and self-discharge power. BRate $_{B E S S i}^{\text {Char }}(t)$ and BRate $_{B E S S i}^{\text {Dischar }}(t)$ denote the charge and discharge rate of the $i$ th BESS. To encourage BESS to participate in the optimal scheduling process, its discharging price is higher than that of the charging price, in which the former is regarded as revenue and the later is treated as cost. Equations (15), (16), and (18) show that charge power, discharge power, and current capacity of the $i$ th BESS are limited in their lower and upper bounds. Equation (17) gives the state equation of capacity for the $i$ th BESS.

6) Power exchange constraints

LEN can exchange power with its neighbor LEN and/or connected upper node of LENs. If production exceeds its requirement, the redundant part of LEN will be sought by its neighbor LEN and the connected upper node of LENs, successively. If demand exceeds supply, LEN will first purchase power to offset its insufficient part from its neighbor LEN, and then from its connected upper node of LENs. The power exchange between LEN and its external grids are restricted by the lower and upper bounds of the transmission lines. We assume that each LEN can exchange power with its multiple neighbor LEN and only one connected upper node, by which the exchange amount can satisfy its internal supply and demand requirements. In order to use DRES, the internal exchange price of LEN is set as RTP, which is lower than the buying price from its neighbor LEN as well as connected upper node and higher than the selling price to them.

$$
\begin{gathered}
C_{\text {NeiLEN }}(t)=\sum_{\text {NeiLENi=1 }}^{\text {NeiLENn }}\left(P_{\text {NeiLENi }}^{\text {Buy }}(t)^{*} \operatorname{price}_{\text {NeiLENi }}^{\text {Buy }}(t)-P_{\text {NeiLENi }}^{\text {Sell }}(t)\right. \\
\left.* \operatorname{price}_{\text {NeiLENi }}^{\text {Buy }}(t)-P_{\text {NeiLENi }}^{\text {Sell }}(t) * \operatorname{price}_{\text {NeiLENi }}^{\text {Sell }}(t)\right)
\end{gathered}
$$


$C_{U p \text { Node }}(t)=P_{U p N o d e}^{\text {Buy }}(t) *$ price $_{U p N o d e}^{B u y}(t)-P_{U p N o d e}^{\text {Sell }}(t) *$ price $_{U p N o d e}^{\text {Sell }}(t)$

$$
\begin{gathered}
P_{\text {NeiLENi }}^{\min }(t) \leq P_{\text {NeiLENi }}(t) \leq P_{\text {NeiLENi }}^{\max }(t) \\
P_{\text {UpNode }}^{\min }(t) \leq P_{\text {UpNode }}(t) \leq P_{\text {UpNode }}^{\max }(t) \\
\operatorname{price}_{R T P}(t)<\text { price }_{\text {NeiLENi }}^{\text {Buy }}(t)<\text { price }_{U p n o d e}^{\text {Buy }}(t) \\
\operatorname{price}_{\text {Upnode }}^{\text {Sell }}(t)<\text { price }_{\text {NeliLENi }}^{\text {Sell }}(t)<\text { price }_{R T P}(t)
\end{gathered}
$$

... where $C_{\text {NeiLEN }}(t)$ and $C_{\text {UpNode }}(t)$ indicate the exchange cost between LEN and its neighbor LEN or connected upper node of LENs. Note that the buying and selling cost are regarded as positive and negative value, respectively. $P_{\text {NeiLENi }}(t)$ and $P_{\text {UpNode }}(t)$ represent the exchange power between LEN and its $i$ th neighbor LEN, as well as the connected upper node of LENs, which are constrained within their lower bound $P_{N e i L E N i}^{\min }(t), P_{U p N o d e}^{\min }(t)$, and upperbound $P_{N e i L E N i}^{\max }(t)$ and $P_{U p N o d e}^{\max }(t)$, respectively. Equation (23) shows how LEN needs to buy power successively from its neighbor LEN and connected upper node of LENs to balance its internal supply and demand requirements. While equation (24) means that the sufficient power of LEN is first sold to its neighbor LEN and then to its connected upper node of LENs.

\section{Optimization Model of LENS}

All the supply and demand requirements of LEN are polymerized to corresponding LENs. DNO of each LENs is responsible for distributing sufficient/insufficient electric power among its controllable LEN, and the abundant or inadequate part of LENs is scheduled by GCC. LENs has self-coordinative ability by conveying operational instructions from GCC to LCC and polymerizing electric power from LCC to GCC. Here the distance-based loss rate and the exchange priority of internal/external nodes are considered. For example, the distance-based loss rate between LEN and its neighbor LEN is lower than that of other LENs, while the usage sequence of DRES production for a certain LEN is first its inner sub-nodes, then its neighbor LEN and, ultimately, its connected upper-level node.

We assume that each LEN has the opportunity to exchange power with other LENs by LCC within the same LENs, but cannot directly exchange power with a LEN located in different a LEN. For each unit electric power of LEN, the buying price from its neighbor LEN is lower than that from its connected upper node, but higher than itself. While the selling price from its neighbor LEN is higher than that from its connected upper node, it is lower than itself. The distribution of sufficient/insufficient electrical power among different LENs is charged by DNO, whose mathematical formulations are evaluated as follows: $\min \operatorname{Cost}_{L E N S}=\sum_{t=1}^{T} \sum_{m=1}^{M} \sum_{n=1}^{N}\left(P(t)_{m n} *\left(C M_{m n}^{H}-C M_{m n}^{L}\right) * \operatorname{Pr} \operatorname{ice}(t)_{m n}\right)$

$$
\left.\begin{array}{c}
P(t)_{11}+P(t)_{12}+, \ldots,+P(t)_{1 n} \leq P(t)_{1}^{\text {Sufficient }} \\
P(t)_{21}+P(t)_{22}+, \ldots,+P(t)_{2 n} \leq P(t)_{2}^{\text {Sufficient }} \\
\cdots \\
\begin{array}{c}
\ldots(t)_{m 1}+P(t)_{m 2}+, \ldots,+P(t)_{m n} \leq P(t)_{M}^{\text {Sufficient }}
\end{array}
\end{array}\right\} M \text { Constraints }
$$

$$
\begin{aligned}
& P(t)_{11}+P(t)_{21}+, \ldots,+P(t)_{m 1} \leq P(t)_{1}^{\text {Insufficient }} \\
& \left.\begin{array}{c}
P(t)_{12}+P(t)_{22}+, \ldots,+P(t)_{m 2} \leq P(t)_{2}^{\text {Insufficient }} \\
\ldots \\
P(t)_{1 n}+P(t)_{2 n}+, \ldots,+P(t)_{m n} \leq P(t)_{N}^{\text {Insufficient }}
\end{array}\right\} N \text { Constraints }
\end{aligned}
$$

$$
\begin{aligned}
P(t)_{1}^{\text {Sufficient }}+ & P(t)_{2}^{\text {Sufficient }}+, \ldots,+P(t)_{M}^{\text {Sufficient }}=P(t)_{1}^{\text {Insufficient }}+ \\
& +P(t)_{2}^{\text {Insufficient }}+, \ldots,+P(t)_{N}^{\text {Insufficient }}
\end{aligned}
$$

$$
0 \leq P(t)_{m n} \leq \min \left(P(t)_{M}^{\text {Sufficient }}, P(t)_{N}^{\text {Insufficient }}\right)
$$

$$
C M_{\mathrm{mn}}^{H}=\left[\begin{array}{ccccc}
\operatorname{Coef}_{11}^{H} & \operatorname{Coef}_{12}^{H} & \operatorname{Coef}_{13}^{H} & \cdots & \operatorname{Coef}_{1 Z}^{H} \\
\operatorname{Coef}_{21}^{H} & \operatorname{Coef}_{22}^{H} & \operatorname{Coef}_{23}^{H} & \cdots & \operatorname{Coef}_{2 Z}^{H} \\
\operatorname{Coff}_{31}^{H} & \operatorname{Coef}_{32}^{H} & \operatorname{Coef}_{33}^{H} & \cdots & \operatorname{Coef}_{3 Z}^{H} \\
\cdots & \cdots & \cdots & \cdots & \cdots \\
\operatorname{Coef}_{Z 1}^{H} & \operatorname{Coef}_{Z 2}^{H} & \operatorname{Coef}_{Z 3}^{H} & \cdots & \operatorname{Coef}_{Z Z}^{H}
\end{array}\right]
$$

$$
C M_{\mathrm{mn}}^{L}=\left[\begin{array}{ccccc}
\operatorname{Coef}_{11}^{L} & \operatorname{Coef}_{12}^{L} & \operatorname{Coef}_{13}^{L} & \cdots & \operatorname{Coef}_{1 Z}^{L} \\
\operatorname{Coef}_{21}^{L} & \operatorname{Coef}_{22}^{L} & \operatorname{Coef}_{23}^{L} & \cdots & \operatorname{Coef}_{2 Z}^{L} \\
\operatorname{Coef}_{31}^{L} & \operatorname{Coef}_{32}^{L} & \operatorname{Coef}_{33}^{L} & \cdots & \operatorname{Coff}_{3 Z}^{L} \\
\cdots & \cdots & \cdots & \cdots & \cdots \\
\operatorname{Coef}_{Z 1}^{L} & \operatorname{Coef}_{Z 2}^{L} & \operatorname{Coef}_{Z 3}^{L} & \cdots & \operatorname{Coef}_{Z Z}^{L}
\end{array}\right]
$$

...where $\operatorname{Cost}_{L E N S}$ means objective value and $P(t)_{m n}$ presents the transmission energy from the $m$ th sufficient node to the $n$th insufficient node at simulation interval $t$. $C M_{m n}^{H}$ and $C M_{m n}^{L}$, shown as equations (30) and (31), respectively, denote the coefficient matrix of the buying and selling LEN, whose elements of the former are larger than 1 and those of the later are smaller than 1 . Price $(t)$ stands for the common reference price of all LENs, which is considered to constitute the practical market clearing price, such as RTP.

Equation (25) gives the objective function, which presents the discounted loss value and classes the participant LENs into two categories: the sufficient LEN and insufficient LEN. The former has superfluous energy output and the later needs to receive some energy from another LEN or its connected upper node to balance its internal supply and demand requirements. The discounted loss value means discrepant line loss and service cost. Note that the state of the connected upper node of LENs is decided by the dispatched requirements of all participating LENs. For example, if the power distribution among all LENs are imbalanced and the surplus amount is positive, 
their connected upper node of LENs need absorb that energy with a relative low power price. Otherwise the connected upper node should provide some energy with relative high power price. So the connected upper node of LENs is regarded as a production node if all the requirements of its controlled LEN are insufficient. Otherwise it is treated as a consumption node to balance the requirements of its governable LEN.

The amount of all the distributed LEN is set as $Z$ during simulation interval $t$, which is classified as $M$-sufficient and $N$-insufficient LEN. For each sufficient LEN, its abundant energy has a different opportunity to be assigned to any insufficient LEN, while the requirements of each insufficient LEN has a different opportunity to get the help of any sufficient LEN. Equations (26) and (27) give the distribution relationship of $M$-sufficient and $N$-insufficient nodes, respectively. Equation (28) shows the equality constraint of all sufficient and insufficient nodes, while equation (29) denotes the lower and upper bound of each decision variable $P(t)_{m n}$ during simulation interval $t$.

\section{Optimization Model of Networked Multiple LENS}

Networked multiple LENs have stronger self-adaption and inter-adjustment ability than that of a single LEN or LENs, which can be illustrated by three aspects. First, limited by the uncertainty of DRES and load requirements, the finite adjustable range of LEN and LENs cannot reach the supply-demand balance state all the time. Secondly, the interconnection among different hierarchical and regional nodes (e.g., LENs or LEN) can enhance their economic scheduling and self-healing abilities, which can partly reduce the burden of networked multiple LENs. Thirdly, networked multiple LENs can make full use of regional cooperation as well as the help from TPP, large-scale EVs, and controllable loads to balance their supply and demand requirements. Though most of the sufficient/insufficient requirements from LEN and/or LENs are compensated for by their internal adjustment and/or exchanging power with neighbor nodes, the remaining parts are accumulated to the level of networked multiple LENs, which has enough production/consumption capacity to offset the accumulated requirements by scheduling its controllable units, such as TPP, EVs, and ILs.

So the optimization process of networked multiple LENs is based on the optimization results of LEN and LENs, in which LENs is treated as the production/ consumption node. The objective function and constraints of networked multiple LENs are not only guaranteeing the co-ordination of all the involved nodes, but also minimizing the operational cost during all the simulation intervals $T$, which are formulated as follows:

$\min \operatorname{Cost}_{M L E N s}=\sum_{t=1}^{T}\left(C_{T P P}(t)+C_{\text {LarEVS }}(t)+C_{\text {LarLoad }}(t)+C_{L E N s}(t)\right)$

$$
\begin{gathered}
C_{T P P}(t)=\sum_{T P P i=1}^{T P P n}\left(\alpha_{T P P i} * P_{T P P i}(t)^{2}+\beta_{T P P i} * P_{T P P i}(t)+\gamma_{T P P i}\right) \\
C_{L a r E V s}(t)=\sum_{E V S i=1}^{E V S n} P_{E V s i} * R C_{E V S i} / \text { ERate }_{E V s i} * \text { price }_{E V S}(t)
\end{gathered}
$$

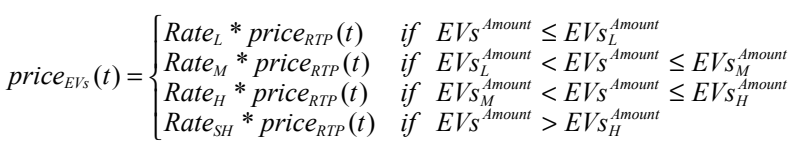

$C_{\text {LarLoad }}(t)=\sum_{\text {LarLoadi }=1}^{\text {LarLoadn }}\left(P_{\text {LarLoadi }}(t) *\right.$ Rate $_{\text {LarLoadi }}(t) *$ price $\left._{\text {RTP }}(t)\right)$

$C_{L E N S}(t)=\sum_{L E N S i=1}^{L E N s n}\left(\left(P_{L E N i}^{B u y}(t) * \operatorname{Rate}_{L E N S i}^{B u y}(t)-P_{L E N i}^{S e l l}(t) * \operatorname{Rate}_{L E N S i}^{\text {Sell }}(t)\right) *\right.$ price $\left._{R T P}(t)\right)$

$$
\begin{aligned}
& P_{L E N s i}(t)= \begin{cases}P_{L E N s i}^{B u y}(t) & \text { if } P_{L E N s i}(t) \geq 0 \\
P_{L E N s i}^{S e l l}(t) & \text { if } P_{L E N s i}(t)<0\end{cases} \\
& P_{T P P i}^{\min }(t) \leq P_{T P P i}(t) \leq P_{T P P i}^{\max }(t) \\
& P_{E V s i}^{\min } \leq P_{E V s i} \leq P_{E V s i}^{\max } \\
& P_{\text {LarLoadi }}^{\min }(t) \leq P_{\text {LarLoadi }}(t) \leq P_{\text {LarLoadi }}^{\max }(t) \\
& P_{L E N s i}^{B u \min }(t) \leq P_{L E N S i}^{B u y}(t) \leq P_{L E N S i}^{B u y \max }(t) \\
& P_{L E N s i}^{\text {Sell min }}(t) \leq P_{L E N s i}^{\text {Sell }}(t) \leq P_{L E N s i}^{\text {Sell max }}(t) \\
& P_{T P P i}(t)+P_{L E N s i}^{B u y}(t)=P_{\text {LarLoadi }}(t)+P_{E V S i}(t) / \text { ERate }_{E V s i}+P_{L E N S i}^{\text {Sell }}(t)
\end{aligned}
$$

Equation (32) provides the objective function for the third stage of our proposed framework, in which Cost $_{M L E N S}$ means the total cost of multiple LENs during all the simulation intervals $T . C_{T P P}(t), C_{\text {LarEVS }}(t), C_{\text {LarLoad }}(t)$, and $C_{L E N s}(t)$ stand for the cost of TPP and large-scale EVs and loads, as well as LENs during simulation interval $t$, respectively. Among them, the relationship of $\operatorname{cost} C_{T P P}(t)$ and output power $P_{T P{ }_{i}}(t)$ for TPP is expressed by a quadratic equation, (33), where $\alpha_{T P P i}, \beta_{T P P i}$, and $\gamma_{T P P i}$ denote the quadratic, monomial, and constant coefficient of the $i$ th TPP. Note that the production cost of TPP is changeable with different output power $P_{T P P}(t)$. Equation (34) shows the cost of large-scale EVs, in which $P_{E V S i}$ presents the amount of EVs, $R C_{E V S i}$ means the rated capacity of each EVs, and ERate ${ }_{E V S i}$ stands for the transformation rate of the $i$ th EVs. price ${ }_{E V S i}(t)$ presents the charge price of EVs, 
which is decided by RTP and the amount of EVs, shown as equation (35). The cost of large-scale loads $C_{\text {LarLaad }}(t)$ is expressed by equation (36), which is a negative value and regarded as revenue. Rate $_{\text {LarLoad }}(t)$ denotes the loss rate of transmission lines as well as transformation efficiency. Equations (37) and (38) show the cost $C_{L E N S}(t)$ and output power $P_{L E N S i}(t)$ of networked multiple LENs, which means that each LENs needs to exchange its sufficient/ insufficient power energy with other LENs. The positive $P_{\text {LENsi }}(t)$ means the production of the $i$ th LENs is sufficient and it should sell superfluous part $P_{L E N S i}^{\text {Sell }}(t)$ with a lower price than RTP, while the negative $P_{L E N S i}(t)$ represents the demand of the $i$ th LENs exceeding its supply and it should buy corresponding power energy $P_{L E N s i}^{B u y}(t)$ to compensate for its insufficient part. $\operatorname{Rate}_{L E N S i}^{B u y}(t)$ and Rate $_{\text {LENSi }}^{\text {Sell }}(t)$ present the exchange loss between LENs and its external grids. Equations (39)-(43) show the lower and upper constraints for the output power of TPP $P_{T P P i}^{C h a r}(t)$, the amount of EVs $P_{E V S}$, the input power of large-scale loads $P_{\text {LorLoadi }}(t)$, the exchanged power of LENs $P_{L E N S i}^{B u y}(t)$, and $P_{L E N S i}^{\text {Sel }}(t)$, respectively. The power balance constraint of networked multiple LENs is given by equation (44).

\section{Optimization Process and Methodology}

\section{Optimization Process}

LENs constitute the skeleton of EI, which contributes to making full use of DRES anytime and anywhere. In each LENs, the exchange information and power flows are bi-directionally and independently implemented for all LEN at the first stage. If buying/selling requirements still exist after the first stage, the remainder requirements are polymerized to DNO - all of which are regarded as scheduled resources to participate in the optimization process of LENs at the second stage. Then the requirements of all LENs together with other units (e.g., TPP, largescale EVs, and loads) are optimized at the third stage. The flowchart of the above three stages is shown as Fig. 3 .

1. The first stage shows the process of parameter initialization and optimization for all LEN. First, the parameters of generation, consumption, BESS, and connected upper nodes for each LEN are initialized. Among them, the output power of controllable/ uncontrollable generators (e.g., NGG, WDG, and PVG) are included in the generation side. The consumption side contains two types of loads, named CLs and ILs. BESS is in the charging state when RTP is low or supply exceeds demand; otherwise, BESS is in the discharge state. At this stage, other parameters, such as population size, maximum iteration times, and the amount of LEN are also initialized. Secondly, if imbalance still exists after internal exchange of LEN, its neighbor and/or connected upper nodes are used to participate in the process of offsetting the sufficient/ insufficient requirements. After several iterations, the terminal condition is met and the optimized results are ultimately output, which are regarded as the input parameters of the next stage.

2. The second stage gives the optimization process within each LENs. At this stage, all the interconnected LEN belong to the same LENs, which have different energy requirements. For each LEN, its ultimate goal is to reach the balanced state by managing its internal schedulable resources and exchanging power with neighbor and/or connected upper nodes, in which the LEN with positive requirements are regarded as production nodes instead of the consumption nodes. Then the transmission power from each production node to any consumption node is set as a decision variable. For example, if the $n$ LEN is grouped into $n / 2$ production nodes and $n / 2$ consumption nodes, the amount of decision variables is $n / 2 * n / 2$. All the production and consumption nodes are interconnected by the public transmission and distribution lines. If the line lose is not considered, each LEN has an equal opportunity to transmit or receive equivalent power. For distinguishing different transaction situations, the distance-based loss rate is considered in our paper, which can avoid long-distance power transmission and

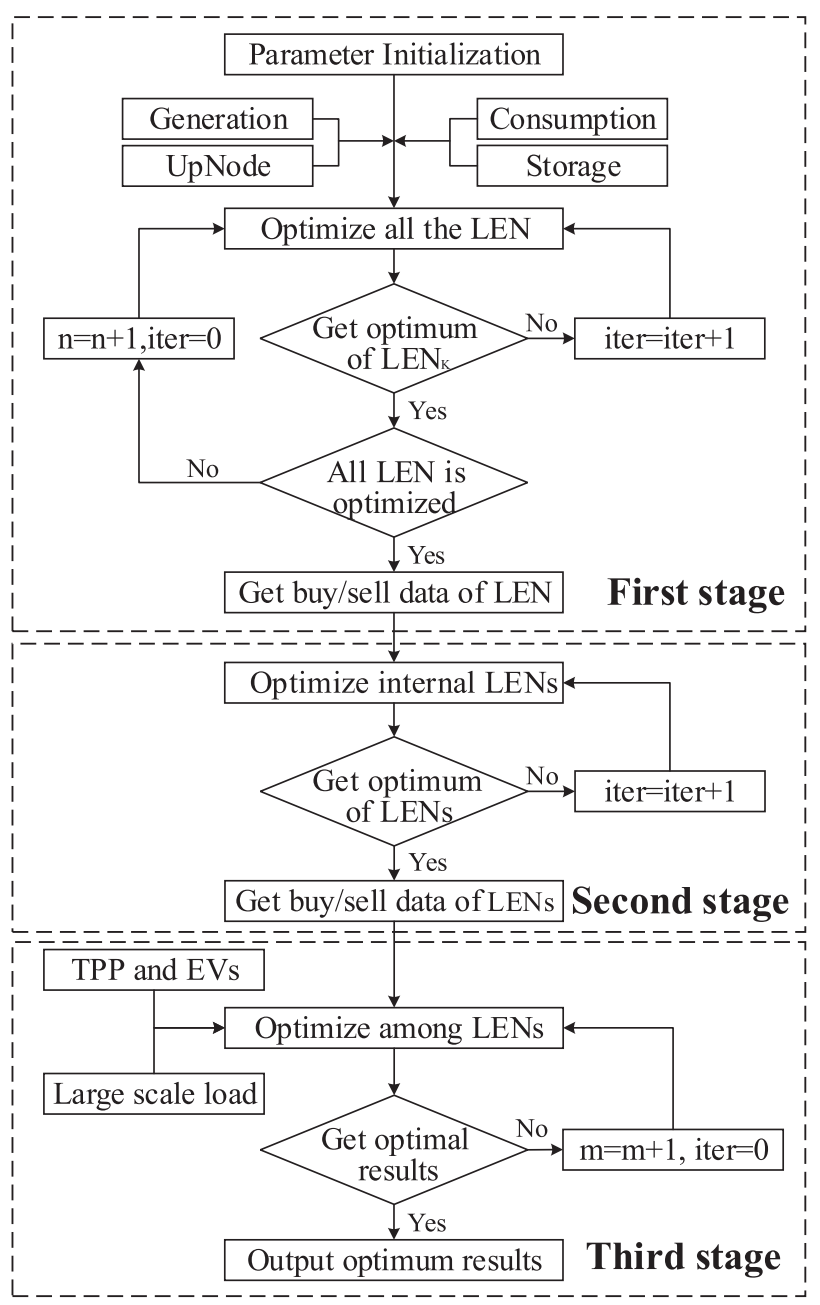

Fig. 3. The flowchart of distribution process for networked multiple LENs in grid-connected mode. 
contribute to making DRES consumed locally. The optimization process of different LENs is parallel and their output results are used to execute the next stage.

3. The third stage is used to optimize the output results of networked multiple LENs as well as other units, such as TPP, large-scale EVs, and loads. The second stage has answered such question about how to distribute the sufficient or insufficient energy among LEN and their neighbor LEN or connected upper nodes, which also output the sufficient/insufficient requirements from each DNO to GCC. GCC is in charge of collecting all the supply-demand information and dispatching the schedulable resources to its controllable nodes, whose optimization objective is not only to balance the supply and demand requirements, but also to minimize the cost of the whole networked multiple LENs at each simulation interval $t$. Due to the fluctuation character of DRES and the finite self-balancing ability of LENs, the output power of TPP, the amount of EVs, and the interrupted ratio of large-scale loads are adjustable and GCC has enough ability to balance all the sufficient and/or insufficient requirements at this stage. Note that the networked multiple LENs can exchange power with the utility grid, which is not discussed in this paper.

\section{Optimization Methodology}

The amount of decision variables produced by the above three stages can reach up to a thousand or even more, which belong to different hierarchies and regions. The traditional optimization methods, such as the multiplier method or gradient-based method are not suitable or highefficiency alone, which need combine other strategies such as evolutionary algorithm.

Considering that the lower and upper bounds of each decision variable are given and other constraints are easily transformed into an unconstrained optimization problem by Lagrange multiplier method (see www.pudn.com), we regard such issue as an LSGO problem and it can be efficiently solved by some evolutionary algorithms, especially when the dimension of decision variables is larger than 1,000. LSGO is an efficient method for dealing with many decision variables, which can support to solve many environmental matters as well as other practical problems, especially for evaluating environmental pollution, reducing carbon emissions, and optimizing the application of renewable energy resources. Moreover, its corresponding codes can be freely obtained and used for academic research.

1) Large-scale global optimization (LSGO) problem. LSGO is a widely used framework to solve the unconstrained optimization problem [26], which adopts evolutionary algorithm as a solver and can rapidly find near-optima solution. The number of decision variables to be optimized for the LSGO problem can reach up to 1,000 or more, whose general formulation can be expressed as follows:

$$
\begin{gathered}
\min f\left(x_{1}, x_{2}, \ldots, x_{i}, \ldots, x_{n}\right) \\
\text { s.t. } \quad x_{\min } \leq x_{i} \leq x_{\text {max }} \quad i \in[1,2, \ldots, n]
\end{gathered}
$$

... where $f(\mathrm{x})$ denotes the objective function and $n$ presents the amount of decision variables. $x_{\min }$ and $x_{\max }$ stand for the lower and upper bounds of each decision variable $x_{i}$.

For solving such an LSGO problem, two typical methods have been widely discussed: the groupingbased method and non-grouping method [27]. The former method is applied for high-dimensional LSGO problems and classifies all the decision variables into several supgroups by some grouping methods, such as the random grouping method [28-29], delta grouping method [30], and differential grouping methods [31-32]. Then the decision variables located in different sup-groups are optimized cooperatively and their results are combined into the ultimate solution. The non-grouping method means all the decision variables are optimized directly and needs a more competitive solver, whose optimization results are better than those of the former method.

2) Optimization method based on LSGO

In order to solve the above optimization problem of networked multiple LENs in grid-connected mode, we propose a novel algorithm framework based on LSGO, which firstly transforms the above optimization problem into an unconstrained optimization problem by Lagrange multiplier method [33], then the self-adaptive differential evolution with neighborhood search (SaNSDE) [34] is employed as a solver to optimize the LSGO problem directly. The specific algorithm is shown as follows:

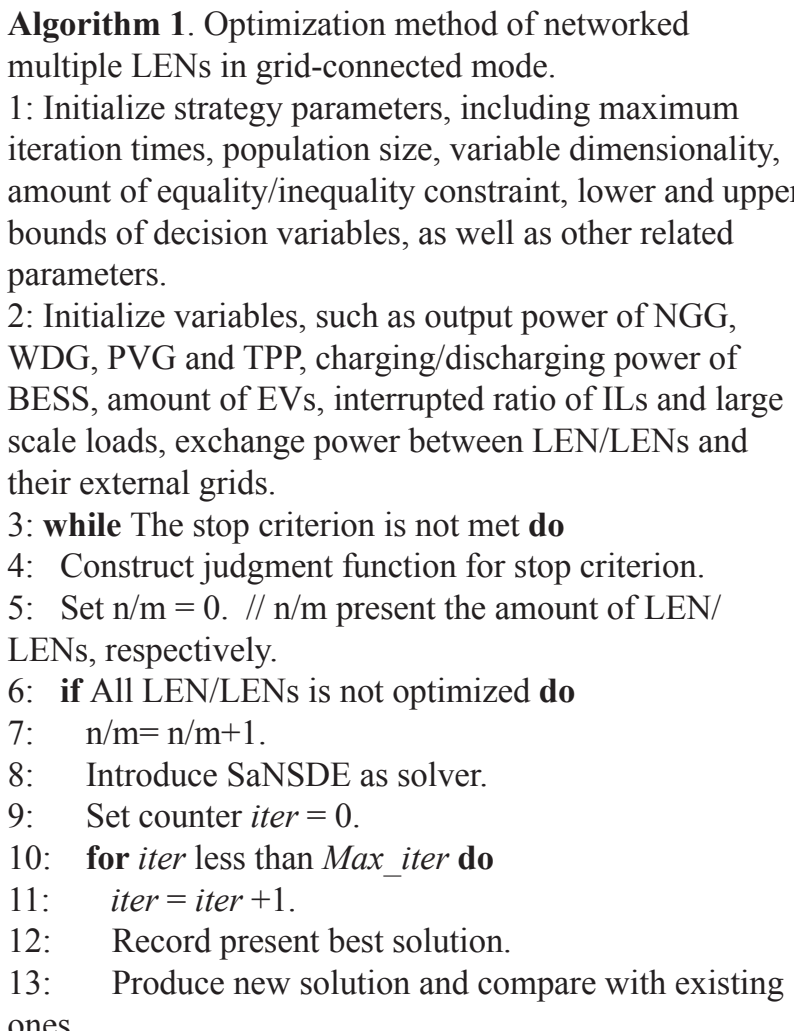
ones. 
14: if New solution is better than existing ones do

15: $\quad$ Replace existing ones by new solution.

16: end if

17: Update present best solution.

18: end for

19: end if

20: Judge whether the stop criterion is met.

21: Update Lagrange multiplier.

22: end while

23: return optimization results.

In order to test the validity and efficiency of our proposed optimization method, 10 constrained functions applied in [35] are selected. The main experimental parameters are set as: both the iteration times of each test constraint function and maximum function evaluation times (FEs) of each iteration, as well as the population size are equal to 50 . The initial iteration point and Lagrange multiplier are set as $N_{i t e r}$ and $N_{\text {Lag }}$, where the former equals the dimensionality of decision variables and the later equals to the amount of equality and inequality constraints. Their optimal values, number of variables, type of function, and the amount of equality/inequality constraints together with experimental results are summarized in Table 1.

As shown in Table 1, the last six columns denote the basic characteristics of 10 constrained functions, in which LI, NI, and NE stand for the amount of linear/nonlinear inequality and nonlinear equality constraint, respectively. The second column gives the optimized results by our proposed method, which indicates that our proposed algorithm is beneficial and reliable. Both the experimental and optimal results of $F_{1}-F_{9}$ are compared and shown in Figs 4a-i), which also illustrate that the optimization algorithm presented in this paper can rapidly and accurately find the optimal value of the tested constrained functions.

\section{Results and Discussion}

In this section, a case study is proposed to verify our proposed method, shown as Fig. 5, which includes three vertical levels.

1. The bottom level is composed of $18 \mathrm{LEN}$, which are marked as LEN A1-A3, B1-B6, and C1-C9, respectively. Each LEN contains a large number of controllable/uncontrollable production units (e.g., NGG, WDG, and PVG), consumption units (e.g., CLs and ILs), and BESS units, which are connected by bus line. For simplification, each type of unit is coupled before interacting with others. The optimization objective of each LEN is to minimize the cost of NGG, WDG, PVG, CLs, ILs, and BESS, as well as the exchange cost with its neighbor and connected upper node of LENs. Different LEN polymerize their requirements into corresponding LENs, such as LENs A, LENs B, and LENs C. For each LENs, the exchange cost between LEN and its neighbor LEN is lower than that with other LEN or its connected upper node.
2. The middle level groups all the 18 LEN into three subgroups, named LENs A, LENs B, and LENs C. For each sub-group, LCC is in charge of the internal power exchange of each LEN and sends the polymerized supply-demand requirements to the point of common coupling (PCC), which is connected with DNO. Note that LCC located in different sub-groups have no direct power or information exchange, which needs the help of adjacent ER and EMS, respectively. The optimization objective of LENs is to satisfy all the requirements from its controllable LEN and minimize the transmission cost from the exporting nodes to the importing nodes during the simulation interval $T$. After balancing internal requirements, the remainder sufficient/insufficient requirements of each LENs are treated as available resources, which are scheduled by GCC to exchange power with TPP, large-scale EVs, and loads.

3. The top level regards the remainder requirements of LENs A-C, production capacity of TPP, and consumption requirements of large-scale EVs/loads as schedulable resources. ER and EMS are employed to exchange power and information between GCC and other regional controllers, e.g., DNO and TPP. We assume that TPP, large-scale EVs, and loads have enough adjustable ability to guarantee GCC to balance the ultimate supply-demand requirements. The optimization objective of the top level is not only to balance the ultimate supply-demand requirements of LENs and other components, but also to minimize the exchange cost.

The main experiment parameters are summarized as follows: the initial input power of PVG and WDG loads, as well as RTP are referred to in [36-37]. For WDG and PVG, their initial construction costs are \$1 million U.S. and $\$ 800,000$, service lives are 50 years and 30 years, wages of workers per year are $\$ 20,000$, rated capacities are $150 \mathrm{MW}$ and $100 \mathrm{MW}$, and operational costs are $\$ 5 / \mathrm{MW}$ and $\$ 3 / \mathrm{MW}$, respectively. The power price of CLs is 1.3 times that of RTP and the compensation prices of ILs are 1.1, 1.3, and 1.5 times those of RTP, which depends on the interrupted ratio of ILs. Both the charge and discharge efficiency of BESS are set as $95 \%$ and the maximum discharge rate is 0.8 times its rated capacity. The initial construction cost of each BESS is $\$ 100,000$ and it can charge/discharge 1e6 times during service life. For each LEN and LENs, the buying price from its connected upper node is 1.5 times that of RTP and the selling price is 0.6 times that of RTP. The simulation period is 24 hours and our proposed method is implemented on one-hour time slots.

\section{Optimizing Results of LEN}

18 LEN are applied and optimized in the first optimization stage for a 24-hour horizon, whose decision variables reach up to 1,296 and are optimized simultaneously. Each LEN operates independently and its optimization objective is how to schedule internal 
Table 1. Optimized results of our proposed algorithm for 10 constraint functions.

\begin{tabular}{|c|c|c|c|c|c|c|c|}
\hline Fun & Results & Optimal & $\begin{array}{c}\text { Num of } \\
\text { variables }\end{array}$ & $\begin{array}{c}\text { Type of } \\
\text { function }\end{array}$ & LI & NE & NI \\
\hline$F_{1}$ & -15.000 & -15.000 & 13 & Quadratic & 9 & 0 & 0 \\
\hline$F_{2}$ & -0.805214 & -0.803619 & 20 & Nonlinear & 1 & 0 & 1 \\
\hline$F_{3}$ & $-1.1949 \mathrm{e}-82$ & -1.000 & 10 & Nonlinear & 0 & 1 & 0 \\
\hline$F_{4}$ & -30665.539 & -30665.539 & 5 & Quadratic & 0 & 0 & 6 \\
\hline$F_{5}$ & -6961.814 & -6961.814 & 2 & Nonlinear & 0 & 0 & 2 \\
\hline$F_{6}$ & 24.147 & 24.306 & 10 & Quadratic & 3 & 0 & 5 \\
\hline$F_{7}$ & -0.095825 & -0.095825 & 2 & Nonlinear & 0 & 0 & 2 \\
\hline$F_{8}$ & 680.368 & 680.630 & 7 & Nonlinear & 0 & 0 & 4 \\
\hline$F_{9}$ & 0.750 & 0.750 & 2 & Quadratic & 0 & 1 & 0 \\
\hline$F_{10}$ & -1.000 & -1.000 & 3 & Quadratic & 0 & 0 & $9^{3}$ \\
\hline
\end{tabular}

a)

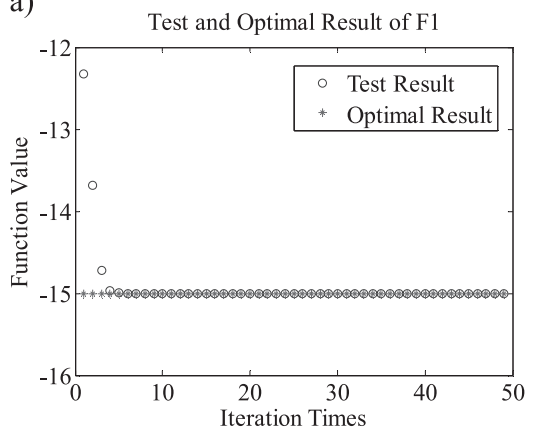

d)
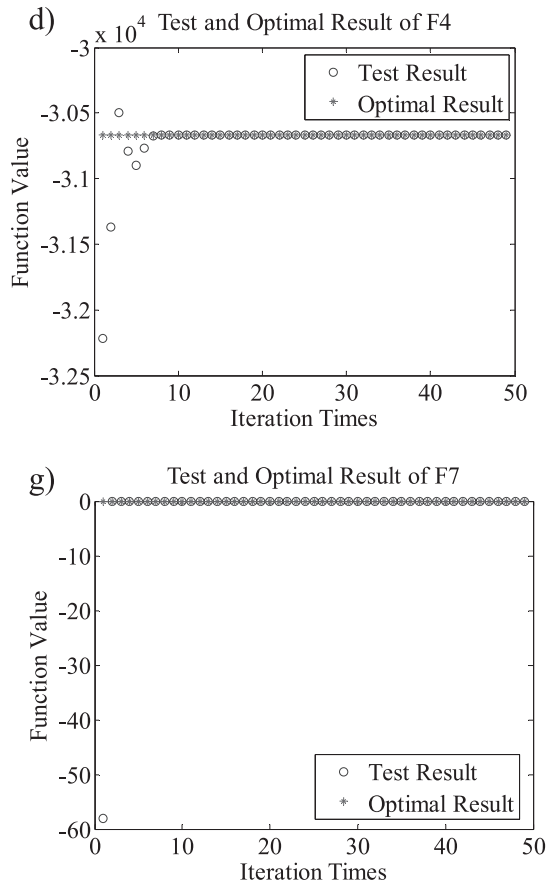

b)
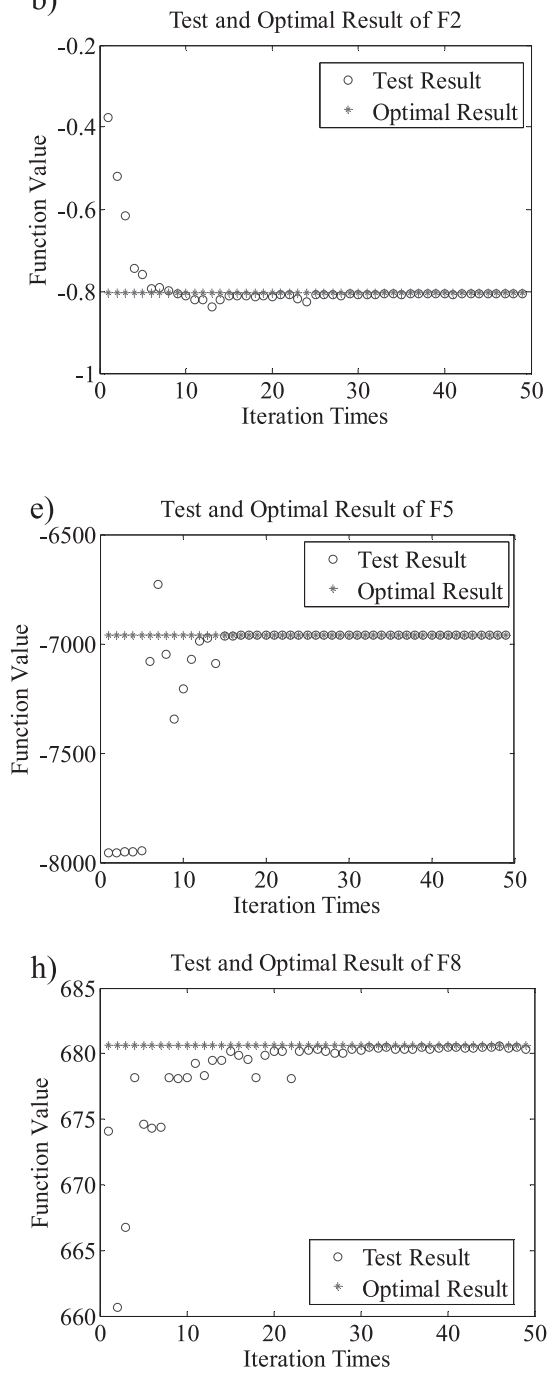

c)

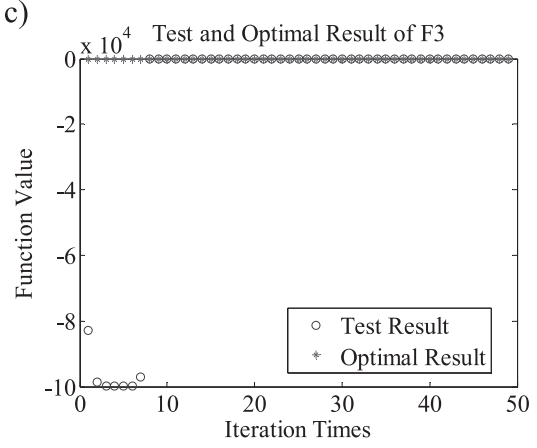

f)

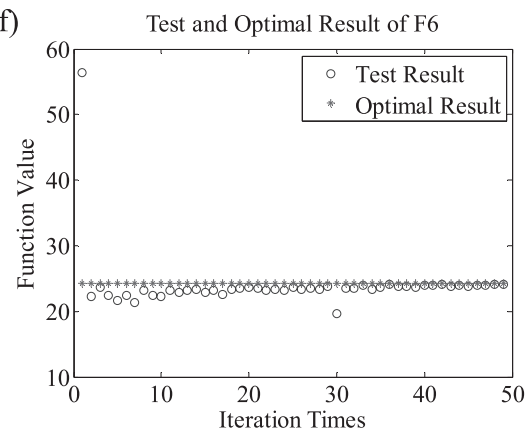

i)

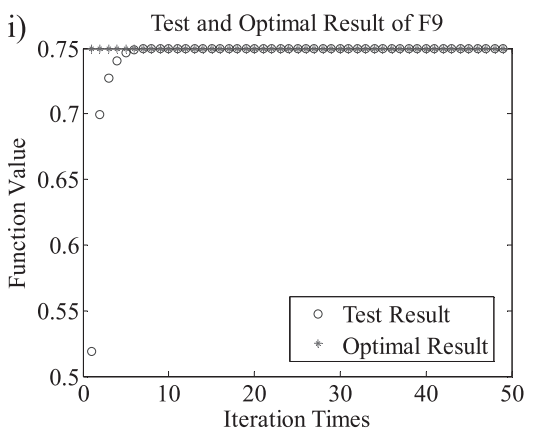

Fig. 4. Optimized results achieved by our proposed method of experimental constrained functions. 


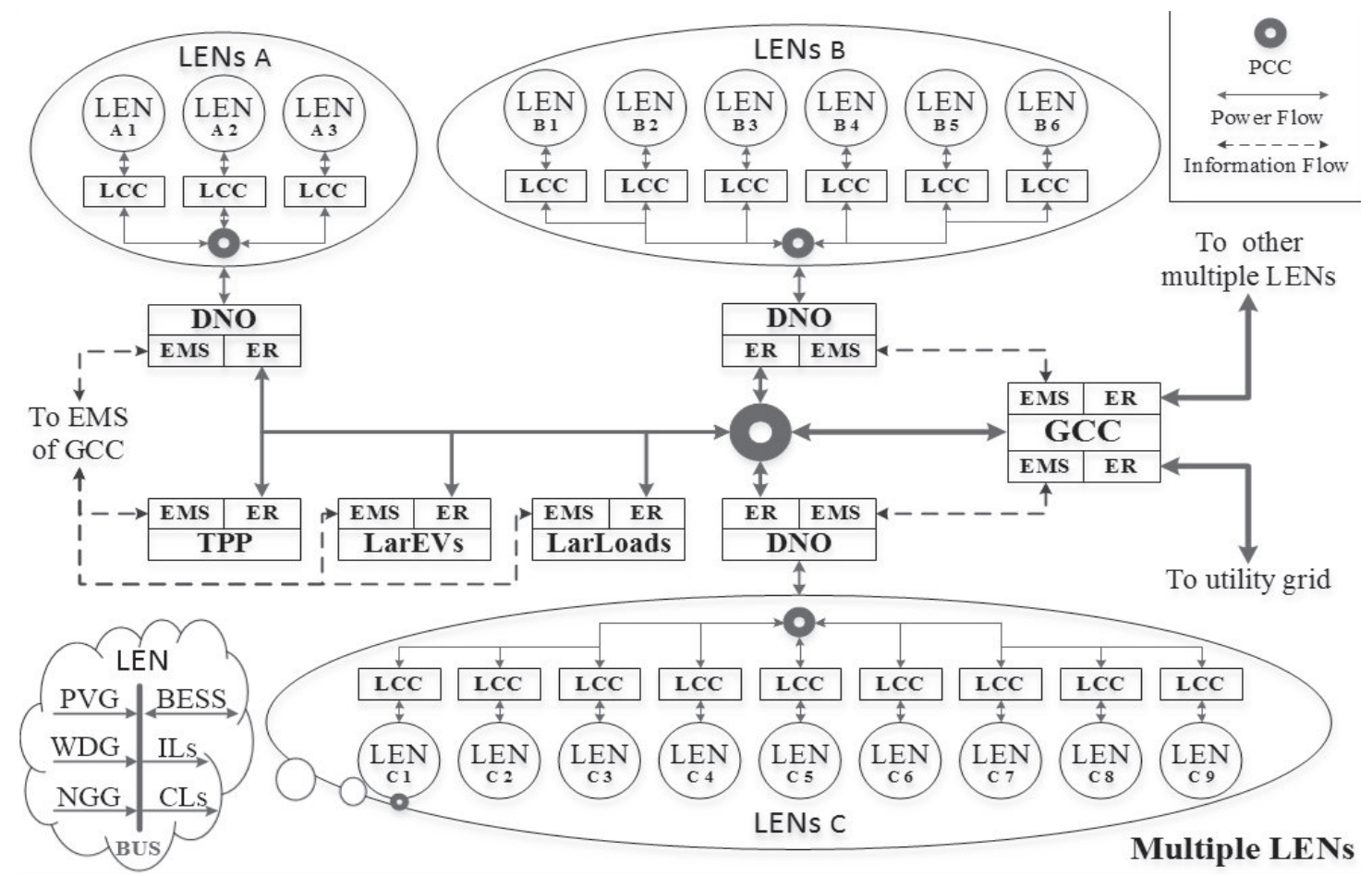

Fig. 5. Three-level optimization framework of networked multiple LENs in grid-connected mode.

controllable units to minimize the operational cost by equations (1)-(24). Taking LEN C9, for example, the optimization results of its internal units are shown as Fig. 6.

Figs (6a-f) show the distribution of optimal output power, average cost of total, and each simulation horizon of generation units, which include NGG, WDG, and PVG. The output power of NGG approaches its rated power (40MW) from 6:00 to the end of the simulation. During such time, its average output cost $(\$ 67.739 / \mathrm{MW})$ for each simulation horizon is lower than that of the total average cost $(\$ 68.028 / \mathrm{MW})$. Its transformation efficiency is located in the high-level $\eta_{N G G}^{H}$ and its maximum practice output power is $39.2 \mathrm{MW}$, indicating that more production quantity of NGG can reach better economic effectiveness by reducing operational cost and increasing transformation efficiency. The experiment result at simulation interval 4 shows a disproof of the above conclusion, where the output power of NGG is 13.698 MW and corresponding average output cost reaches up to $\$ 75.834 / \mathrm{MW}$. Figs $6(\mathrm{~b}, \mathrm{e})$ show the output power and corresponding cost of WDG, in which the fluctuation scope of its output power is from 3.039 MW to 55.548 MW, the corresponding average output cost is $\$ 7.058 / \mathrm{MW}$ and $\$ 5.345 / \mathrm{MW}$, and the total average cost is $\$ 5.661 / \mathrm{MW}$. Similarly, the relationship between the average output cost of WDG and its output power is in inverse proportion. That is, the less output power corresponds to the higher average output cost. The output power of PVG, shown as Fig. 6c), is badly affected by the illumination intensity and its values are zero from 9:00 to 20:00. During such time, the operational cost for each simulation interval $t$ is $\$ 5.327$ and its average output cost is not recorded (shown as a red line in Fig. 6f).

Figs $6(g, j)$ give the charge/discharge power, realtime capacity, and average output cost of BESS. The capacity of BESS is in the lowest situation (1MW) at the beginning and last simulation intervals, and its maximum capacity is $10 \mathrm{MW}$, which fluctuates with the change of charging/discharging processes at other times. The red line with + and the blue line with $*$ mean the real-time charging/discharging power with/without considering the initial construction cost, transforming efficiency, and self-discharge loss, in which the positive parts mean the charging process, the negative values mean the discharging process of BESS, and their absolute values present the charging or discharging amount. Note that the charge/discharge power of BESS is zero at some simulation intervals and the corresponding capacities of BESS are invariable. To encourage the BESS to participate in balancing the internal supply-demand requirements of LEN, its charge price is RTP and the discharging price is three times that of RTP, in which the former is regarded as cost and the later is treated as revenue.

Figs. $6(\mathrm{~h}, \mathrm{k})$ present the total and average output costs of CLs and ILs. In this paper, the power requirements of CLs are satisfied every time and their prices are 1.3 times of RTP. The price of an interrupted part for ILs is grouped into three levels, which are decided by the interrupted ratio LRate $_{I L s i}(t)$ and equal to $1.1,1.3$, and 1.5 times RTP. From Fig. 6h) we see that the ratio of output cost of CLs is smaller than that of ILs, though the unit power price of the former is higher than that of the later. However, the absolute value of average power price of ILs is lower than that of the sum of CLs and ILs, 
which presents the interrupted parts of ILs maintained at a suitable level. The total average power price of ILs (red line with o) together with the sum of ILs and CLs (blue line with o) also show that the ratios of the interrupted part of ILs are small and most of their price are 1.1 times RTP.

Lastly, the exchange power and cost between LEN and its connected upper node are shown as Figs. 6(i,l), which show that LEN C9 belongs to the consumption node and its requirements scope is $[-56.550,0.001]$ MW. The cost of LEN C9 changes with the purchase amount and the exchange price. The total average price between LEN C9 and its connected upper node is \$153.909/MW.

\section{Optimization Results of LENS}

Based on the results of the first stage, the entire 18 LEN have optimized their internal components and output the required amount from/to their connected upper nodes (Fig. 7). At the second stage, there are two strategies to dispatch the input/output requirements among different a)

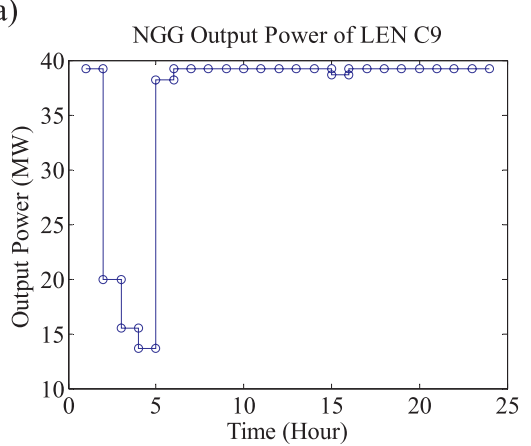

d)

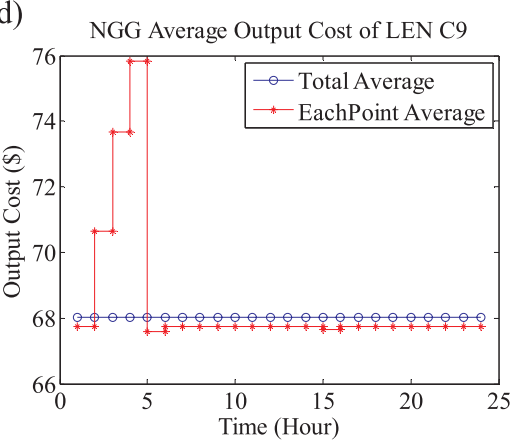

g)

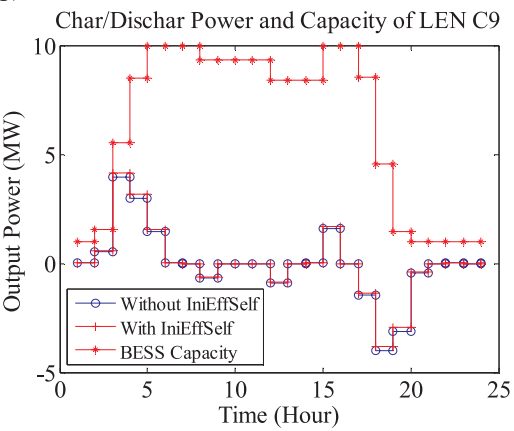

j)

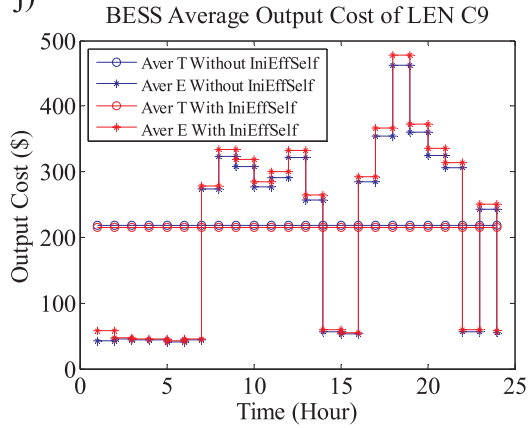

b)

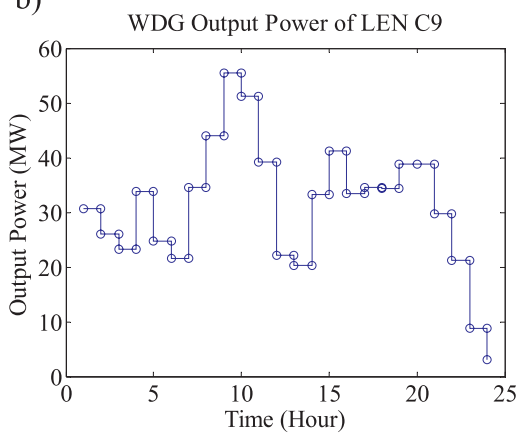

e) WDG Average Output Cost of LEN C9

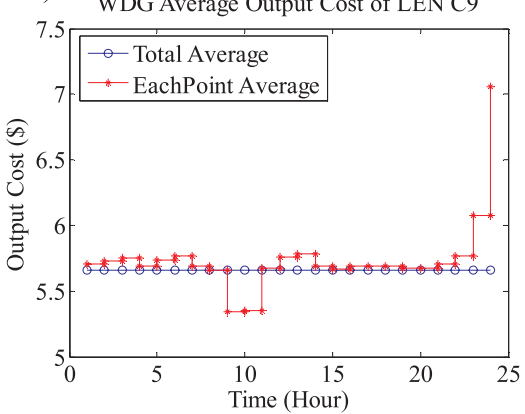

h)

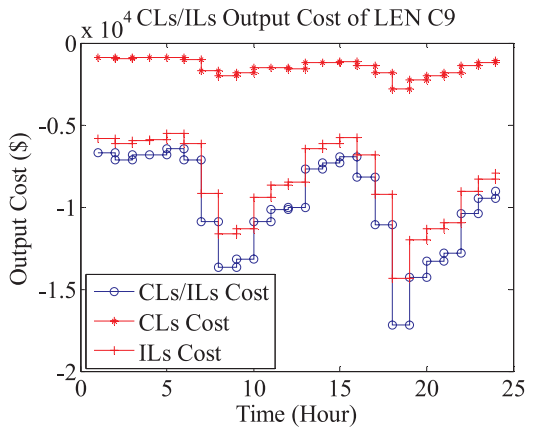

$\mathrm{k})$

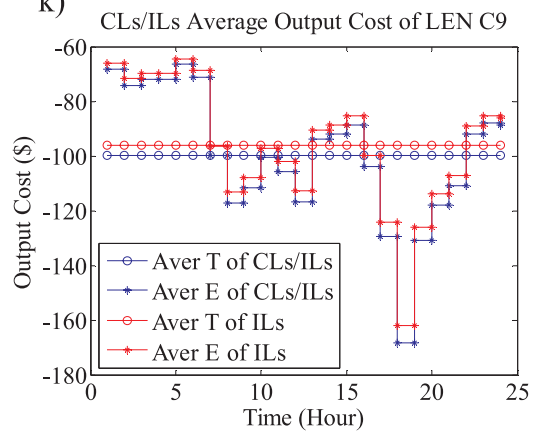

c)

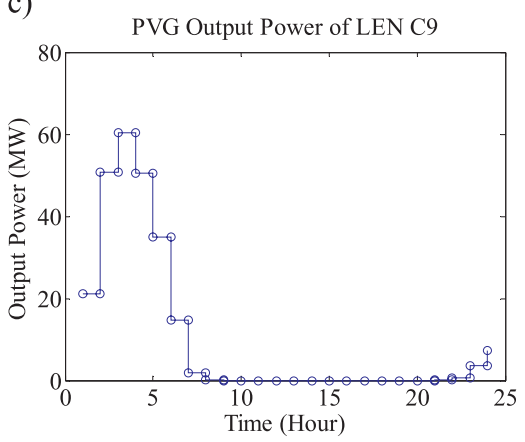

f)

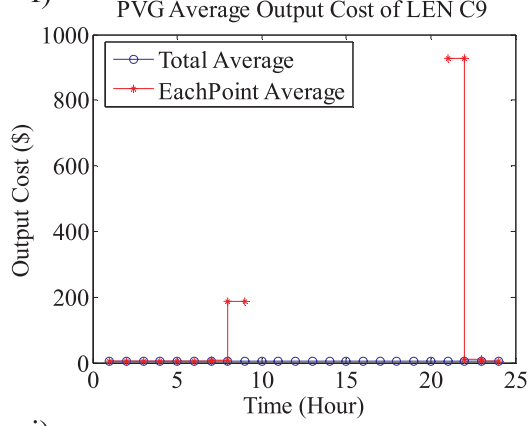

i)

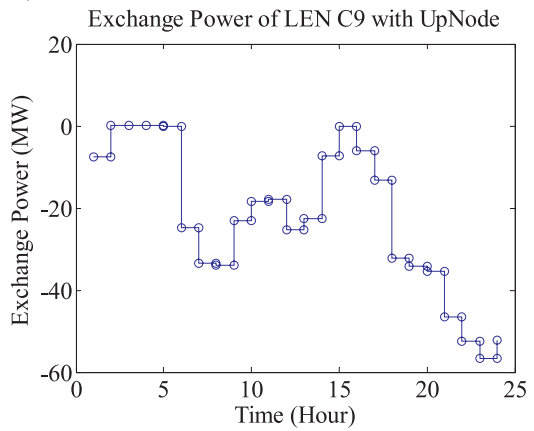

1) Exchange Power Average Output Cost of LEN C9

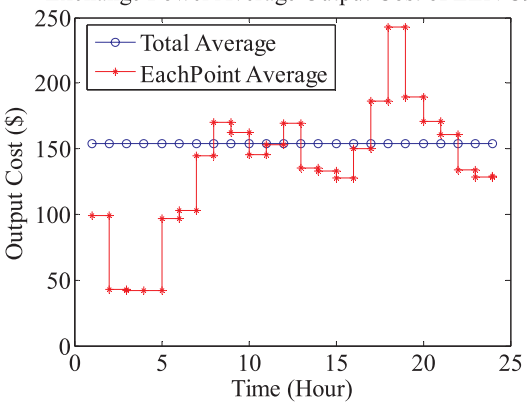

Fig. 6. Optimizing results of LEN (taking C9 as an example). 
LEN and their connected upper nodes: on one hand, each LEN can only exchange power with its connected upper node. On the other hand, each LEN exchanges power with its neighbor LEN firstly, then the redundant or inadequate parts are satisfied by exchanging power with its connected upper node. For realizing this target, the buying price rate of each LEN from its neighbor LEN is lower than that from its connected upper node and is larger than 1, while the selling price rate is opposite and is smaller than 1 . Here we adopt the second strategy and the analysis process is shown as follows:
The sufficient/insufficient requirements of all LEN in the same LENs are first polymerized to respective DNO, and then to their common connected upper nodes. The distribution of total transaction power and cost of LENs A, LENs B, and LEN C are shown as Figs 7(a-c). The exchange costs have no intense regularity with the exchange power, which are caused by the different buying/selling prices. For each LEN, the buying price from its neighbor LEN is lower than that from other LEN or its connected upper node, but higher than RTP, while the selling price to its neighbor LEN is higher than that a)

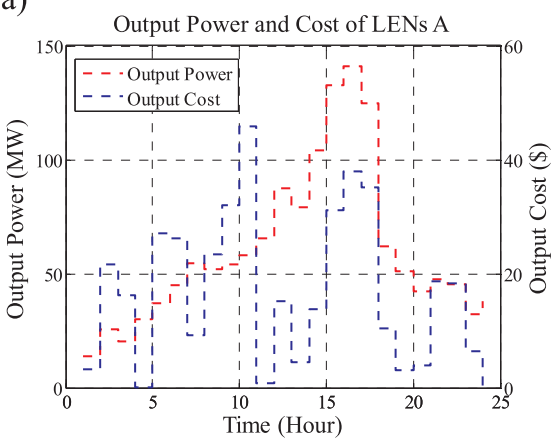

d)

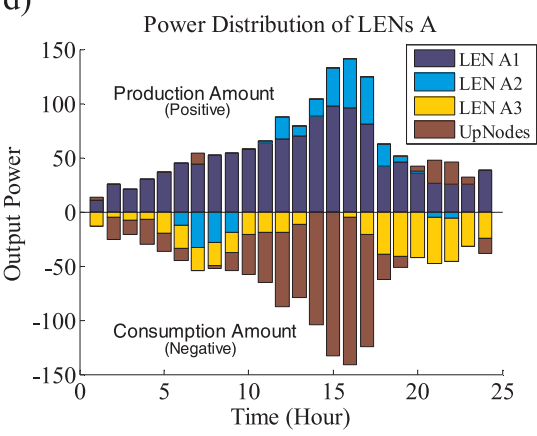

g)

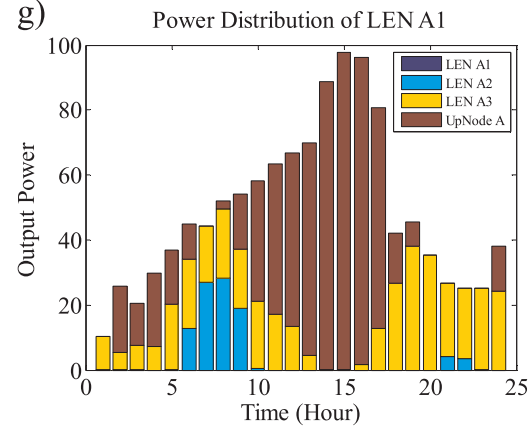

j)

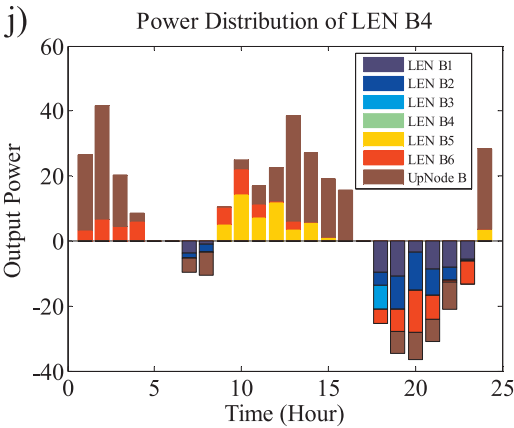

b)

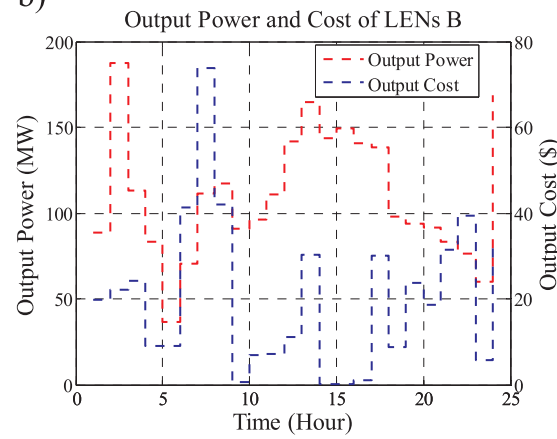

e)

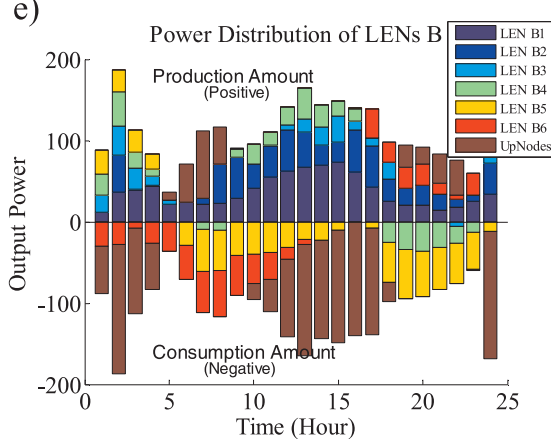

h)

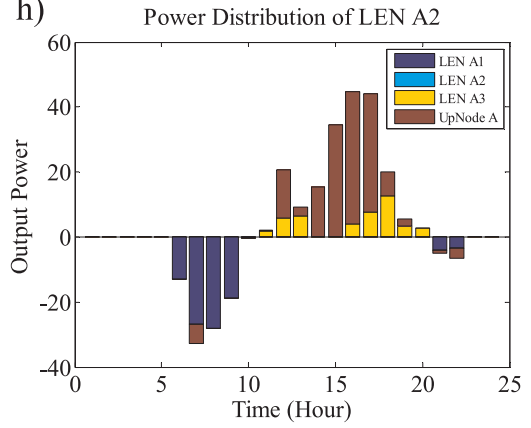

k)

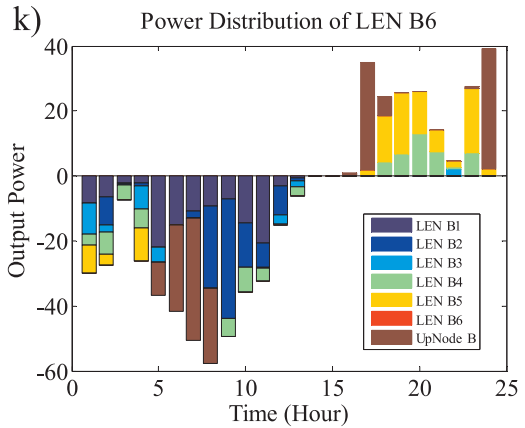

c)

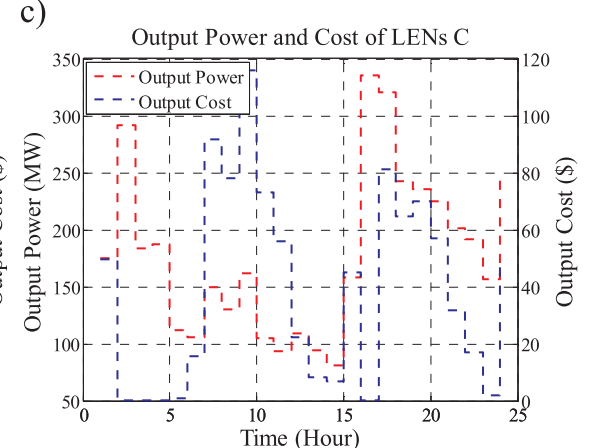

f)

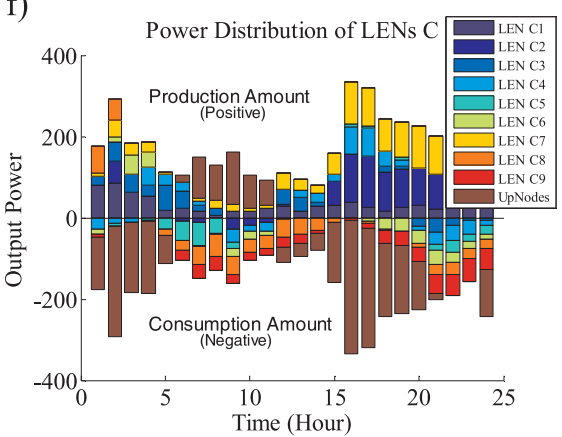

i) Power Distribution of LEN A3

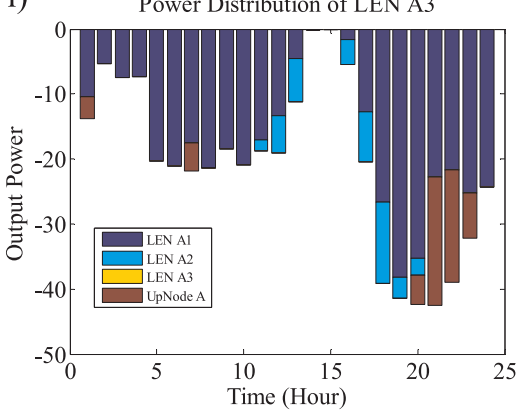

1)

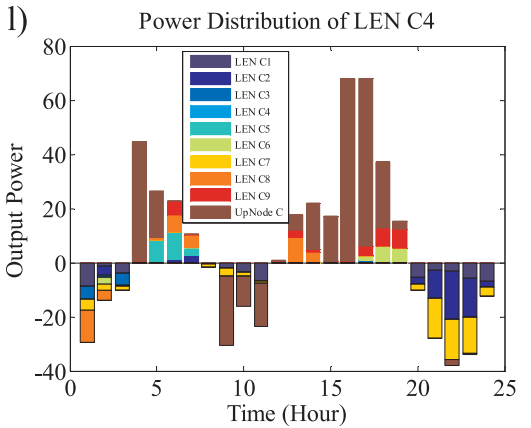

Fig. 7. Optimization results of LENs. 
to other LEN or its connected upper node, but lower than RTP. Specially, the buying price of LEN from its connected upper node is 1.5 times that of RTP and the selling price of that is 0.6 times that of RTP. So the transaction sequence for each LEN is its neighbor LEN firstly, then other LEN and its connected upper node ultimately.

Figs $7(\mathrm{~d}-\mathrm{f})$ show the specific schedulable power of LENs A, LENs B, and LENs C, respectively. All of LEN in the same LENs and their connected upper nodes participate in the power distribution process, in which the positive LEN can output its sufficient power and it is regarded as the production node, while the negative LEN needs to input corresponding power from other nodes and is regarded as the consumption node. If the requirements of consumption nodes cannot be satisfied by their sufficient neighbor nodes, the remainder requirements can be satisfied by other sufficient nodes or even their connected upper nodes, but the buying price will be much higher. For example, the insufficient requirements of LEN A3 is $42.366 \mathrm{MW}$ at simulation interval 20 and it is firstly satisfied by LEN A2 with $2.686 \mathrm{MW}$, then LEN A1 with 35.238 and its connected upper node with 4.442 MW ultimately, in which the buying price of LEN A3 are $1.167,1.333$, and 1.5 times of RTP, respectively.

Then Figs 7(g-i) give more detailed power distribution results of LEN A1, LENA2, and LEN A3, respectively, in which the positive values mean LEN can sell superfluous power to its neighbor LEN or connected upper node of LENs, while the negative values mean LEN should purchase power from its neighboring LEN or connected upper node of LENs. According to the above analysis, LEN A1 can be regarded as a production node, shown as Fig. 7g), and its superfluous powers are dispatched to other nodes every time, especially for LEN A3 and its connected upper node. LEN A3 can be treated as a consumption node and most of its requirements are satisfied by LEN A1 (Fig. 7i). Different from LEN A1 and LEN A3, LEN A2 not only outputs power to LEN A3 and its connected upper node from 11:00 to 20:00, but also inputs power from LEN A1 and its connected upper node at other times (Fig. 7h) and can be regarded as a prosumer node. Meanwhile, LEN A2 can reach self-balance state during some times, such as simulation intervals 1-3.

Then take LEN B4, LEN B6, and LEN C6, for example, shown as Figs 7(j-1), which are also prosumer nodes and taken from LENs B and LENs C, respectively. The power distribution result of LEN B4 is shown as Fig. 7j) and most of its sufficient output power is dispatched to its connected upper node. LEN B4 can reach a selfbalance state at simulation interval 5 and its fluctuation scope is [-36.462,41.684] MW during other simulation times. Fig. 7k) gives the power distribution result of LEN B6, which can be separated into three parts: the former part denotes that LEN B6 should input power from other nodes before 13:00, the middle part shows LEN B6 can also reach self-balance state during simulation interval 14 as well as 15 , and the later part means LEN B6 output its superfluous power to others after 16:00. The total buying and selling power of LEN B6 are
416.285 MW and 197.282 MW, which indicate that LEN B6 need to buy 219.003 MW power from other nodes during all the simulation intervals $T$. Fig. 71) shows the power distribution result of LEN C4, which is regarded as the representation of LENs C. All the simulation results of LEN C4 can be grouped into five sections: two selling sections during 4:00 to 7:00 as well as 12:00 to 19:00, and three buying sections at other times. The total sufficient and insufficient power of LEN C4 are 350.277 MW and 248.096 MW, which indicates that LEN C4 can sell redundant production 102.181 MW to its external nodes, especially for its connected upper node.

\section{Optimization Results of Networked Multiple LENS}

DNO in each LENs polymerizes all the supplydemand requirements of its controllable LEN, and then distributes them with minimum cost. However, there still exists imbalance among LENs and the sufficient/ insufficient requirements of each LENs are transmitted from its DNO to GCC. GCC is responsible for balancing the requirements of networked multiple LENs together with other parts, such as TPP, large-scale EVs, and loads. The optimization results of networked multiple LENs are shown in Fig. 8.

The optimization results of exchange power as well as corresponding cost among LENs and GCC are shown in Fig. 8a), in which the positive values mean GCC needs to input the sufficient powers from LENs by balancing their supply-demand requirements and to afford corresponding cost, while the negative values present GCC can output power to related DNO and obtain corresponding revenue. The total output power from GCC to DNO of all LENs are 657.856 MW during 6:00 to $10: 00$ and 21:00 to 23:00, while all of the input power from DNO to GCC are $4207.437 \mathrm{MW}$ during other times. Meanwhile, the maximum average price of the former is $\$ 102.458 / \mathrm{MW}$, which is much lower than that of the later $\$ 164.169 / \mathrm{MW}$, shown as the bold blue line in Fig. 8a).

The relationship between output power and cost of TPP are proportional (Fig. 8b). The maximum output power of TPP is $400 \mathrm{MW}$ and its practical output scope is $[90.017,374.148] \mathrm{MW}$. The total cost of TPP regularly fluctuates with increasing or decreasing output power, and its practical scope is $\$[486.939,2026.336]$. The minimum average price of output power for TPP is $\$ 46.737 / \mathrm{MW}$ at simulation interval 2 , while its maximum value is \$56.764/MW at simulation interval 12 .

Figs $8(d, e)$ show the consumed power as well as cost of large-scale EVs and loads. GCC can use up the redundant electrical power from LENs to satisfy the requirements of EVs and loads, especially when the scale is very large. The minimum input power of EVs is $0.383 \mathrm{MW}$ at simulation interval 10 (Fig. 8d), which means that five EVs are charging power at such time. While its maximum input power is $636.401 \mathrm{MW}$ at simulation interval 10 and the amount of EVs is 8316. The revenue scope of selling power to EVs is $[44.642,104588.236]$ dollars and the 


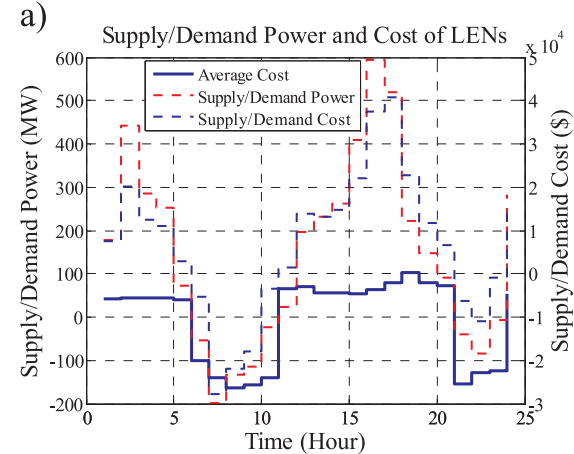

d)

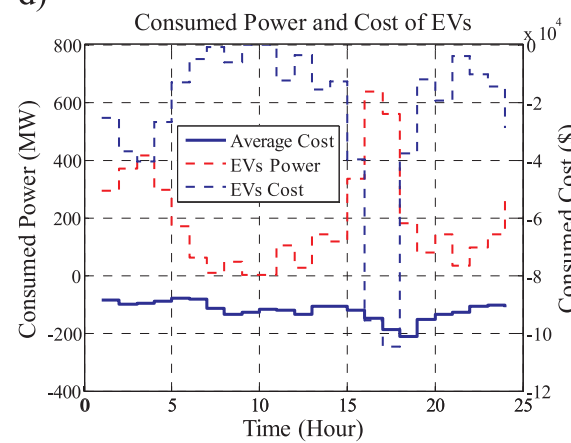

b)

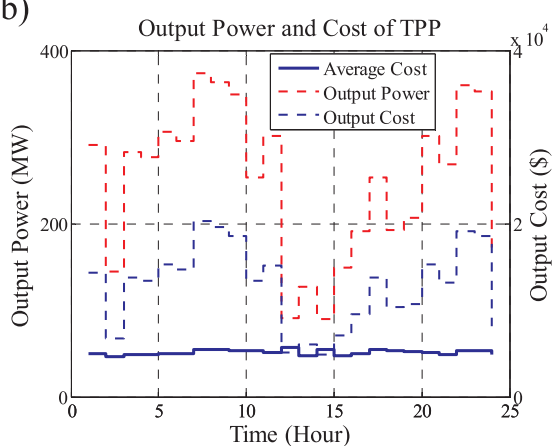

e)

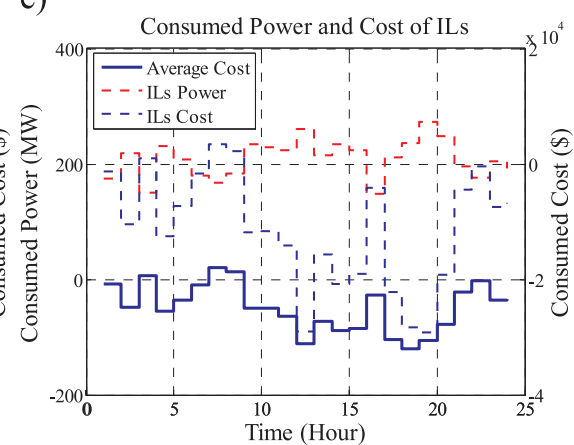

c)

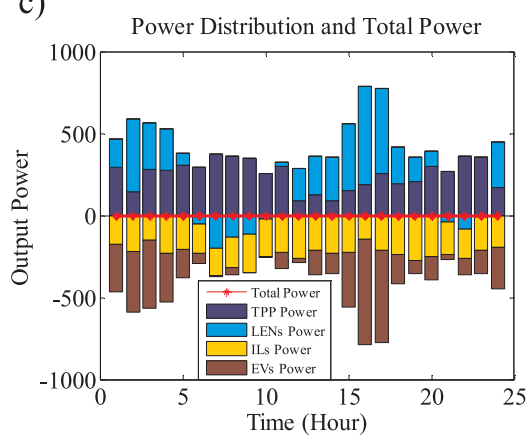

f)

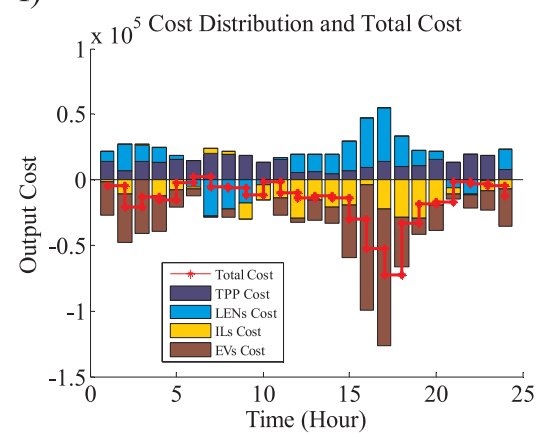

Fig. 8. Optimization results of networked multiple LENs.

total amount are $\$ 567,110.968$ during all the simulation intervals $T$. The average price of EVs changes with their requirements as well as RTP, whose experiment result is $\$[77.472,210.535] / \mathrm{MW}$.

Different from LENs and TPP, the consumed power and cost of large-scale loads have an inversely proportional relationship (Fig. 8e). Note that some of the average prices of large-scale loads are positive, such as $\$ 6.653 / \mathrm{MW}$ at simulation interval 3, because the interrupted ratio of that time is too large (LRate $\left._{I L s i}(t)=0.435\right)$ and the revenue of uninterruptable part is not enough to offset the compensation cost of the interrupted part, so GCC needs to afford corresponding cost to balance the ultimate supply-demand requirements.

Fig. 8c) presents the power distribution of different nodes for networked multiple LENs, in which the positive values mean that corresponding nodes can output power to other nodes and the negative values mean that those nodes need receive power from others. For example, the sufficient power of TPP and LENs are 291.102 MW and 177.722 MW at simulation interval 1, which are transformed to large-scale loads with $174.435 \mathrm{MW}$ and EVs with 294.390MW, respectively. The red line with * in the middle of the figure indicates all the sufficient production amounts at each time justly equal the insufficient requirements of the consumption nodes.

Fig. 8f) gives the cost and revenue distribution of different units for the networked multiple LENs, in which the positive values mean cost and the negative values denote revenue. For example, the cost of buying the insufficient power from TPP and LENs by GCC is $\$ 14,338.159$ and $\$ 7,437.676$ at simulation interval 1 , and the revenue of selling sufficient power to large-scale loads and EVs are $\$ 1,379.470$ and $\$ 25,316.082$, respectively, where GCC can earn $\$ 4,919.717$ after offsetting the cost. Different from the above power distribution, the cost distribution at each simulation interval $t$ is asymmetrical, which is caused by different buying and selling price rates. The total cost of all simulation intervals $T$ for GCC is $-\$ 370,963.531$, shown as the red line with * in the middle of Fig. 8f, which together with Fig. 8c indicates that GCC not only has the ability to balance the ultimate supplydemand requirements, but also obtain abundant economic benefits.

\section{Conclusions}

This paper presents a novel methodology for the optimal power distribution of multiple local energy network systems (LENs) in grid-connected mode. Based on three-level optimal control architectures and six typical operational scenes, the decentralized optimal model of each LEN is first constructed by treating its internal units and connected upper nodes as controllable resources. According to the polymerized sufficient/insufficient requirements from each LEN, DNO is employed by LENs to describe the power dispatching process among sufficient/insufficient nodes of LEN, in which transformation efficiency, transmission loss, and RTP are considered. Then a concentrated optimal model of the top level of our proposed framework is proposed to balance the ultimate supply-demand requirements, in which GCC is responsible for balancing the requirements of networked multiple LENs together with other components, such as TPP, large-scale EVs, and loads. Considering that the 
lower and upper bounds of each decision variable are given and other constraints are easily to be transformed into an unconstrained optimization problem by the Lagrange multiplier method, we regard above optimal control models as an LSGO problem and the self-adaptive differential evolution with neighborhood search method (SaNSDE) is served as a solver. For testing the validity and efficiency of our propose optimization method, ten constrained functions are chosen and the experimental results illustrate that the optimization algorithm presented in this paper can rapidly and accurately find the optimal value. Experiment results of networked multiple LENs show that our proposed method not only truly reflects the development of hierarchical power distribution of multiple LENs, but also rapidly and accurately solves such multilayer constrained problems.

To better solve such problems mentioned above as well as some other practical problems [38-39], more efficient algorithms will be designed in our future work. Secondly, we will consider a power price-based mechanism to coordinate the supply-demand relationship among different LENs. Finally, service cost also needs to be considered to further perfect our model and narrow the gap between theoretical and practical demands.

\section{Acknowledgements}

This work was financially supported in part by the National Science Foundation of China (Nos. 71571187, 71201170, 61403404). We also thank Zhenyu Yang and Xiaodong Li for kindly providing the source code of SaNSDE on the website.

\section{References}

1. FANG X., YANG Q., WANG J., YAN W. Coordinated dispatch in multiple cooperative autonomous islanded microgrids. Applied Energy. 162, 40, 2016.

2. LI, W., ZHANG, H. Decomposition Analysis of Energy Efficiency in China's Beijing-Tianjin-Hebei Region. Polish Journal of Environmental Studies. 26 (1), 189, 2017.

3. JENDRUS, R. Environmental Protection in Industrial Areas and Applying Thermal Analysis to Coal Dumps. Polish Journal of Environmental Studies. 26 (1), 137, 2017.

4. ZHANG Y., LIU B., ZHANG T., GUO B. An Intelligent Control Strategy of Battery Energy Storage System for Microgrid Energy Management under Forecast Uncertainties. Int. J. Electrochem. 9, 4190, 2014

5. WANG Z., CHEN B., WANG J., BEGOVIC M.M., CHEN C. Coordinated Energy Management of Networked Microgrids in Distribution Sysrems. IEEE TRANSACTIONS ON SMART GRID. 6 (1), 45, 2015.

6. WANG Z., CHEN B., WANG J., CHEN C. Networked Microgrids for self-Healing Power Systems. IEEE TRANSACTIONS ON SMART GRID. 7 (1), 310, 2016.

7. BUI V.H., HUSSAIN A., KIM H.M. A Multiagent-Based Hierarchical Energy Management Strategy for MultiMicrogrids Considering Adjustable Power and Demand Response. IEEE TRANSACTIONS ON SMART GRID. DOI 10.1109/TSG.2016.2585671, 2016.
8. XU Y., ZHANG W., HUG G., KAR S., LI Z. Cooperative Control of Distributed Energy Storage Systems in a Microgrid. IEEE TRANSACTIONS ON SMART GRID. 6 (1), 238, 2015.

9. LI Q., PENG C., CHEN M., CHEN F., KANG W., GUERRERO J.M., ABBOTT D. Networked and Distributed Control Method with Optimal Power Dispatch for Islanded Microgrids. IEEE TRANSACTIONS ON INDUSTRIAL ELECTRONICS. DOI 10.1109/TIE.2016.2598799, 2016.

10. CHEN F., CHEN M., LI Q., MENG K., GUERRERO J.M., ABBOTT D. Multiagent-Based Reactive Power Sharing and Control Model for Islanded Microgrids. IEEE TRANSACTIONS ON SUSTAINABLE ENERGY. 7 (3), 1232, 2016

11. MALYSZ P., SIROUSPOUR S., EMADI A. An Optimal Energy Storage Control Strategy for Grid-connected Microgrids. IEEE TRANSACTIONS ON SMART GRID. 5 (4), 1785, 2014.

12. WANG Z., CHEN B., WANG J., KIM J. Decentralized Energy Management System for Networked Microgrids in GridConnected and Islanded Modes. IEEE TRANSACTIONS ON SMART GRID. 7 (2), 1097, 2016.

13. YU X., XU X., CHEN S., WU J., JIA H. A Brief Review to Integrated Energy System and Energy Internet. TRANSACTIONS OF CHINA ELECTROTECHNICAL SOCIETY. 31 (1), 1, 2016.

14. ZHANG T., ZHANG F., ZHANG Y. Study on Energy Management System of Energy Internet. Power System Technology. 40 (1), 146, 2016.

15. ZENG M., YANG Y., LIU D., ZENG B., OUYANG S., LING H., HAN X."Generation-Grid-Load-Storage" Coordinative Optimal Operation Mode of Energy Internet and Key Technologies. Power System Technology. 40 (1), 114, 2016.

16. DOU C., WANG W., HAO D., LI X. MAS-based solution to energy management strategy of distributed generation system. Electrical Power and Energy Systems. 69, 354, 2015.

17. REYASUDIN BASIR KHAN M., JIDIN R., PASUPULETI J.. Multi-agent based distributed control architecture for microgrid energy management and optimization. Energy Conversion and Management. 112, 288, 2016.

18. WANG Y., MAO S., NELMS R.M. On Hierarchical Power Scheduling for the Macrogrid and Cooperative Microgrids. IEEE TRANSACTIONS ON INDUSTRIAL INFORMATICS. 11 (6), 1574, 2015.

19. YANG F., LIU S., WANG H., FANG Y., LI W., ZHENG W. A Decomposition Method to Calculate Available Transfer Capability of Large Interconnected Power Grid. Power System Technology. 38 (1), 138, 2014.

20. MA W.J., WANG J., GUPTA V., CHEN C. Distributed Energy Management for Networked Microgrids Using Online ADMM with Regret. IEEE TRANSACTIONS ON SMART GRID. DOI 10.1109/TSG.2016.2569604, 2016.

21. SUN Q., TENG F., ZHANG H., MA D. Construction of Dynamic Coordinated Optimization Control System for Energy Internet. Proceedings of the CSEE. 35 (14), 3667, 2015.

22. MINCIARDI R., SACILE R. Optimal Control in a Cooperative Network of Smart Power Grids. IEEE SYSTEMS JOURNAL. 6 (1),126,2012.

23. HAMIDI R.J., LIVANI H., HOSSEINIAN S.H., Gharehpetian G.B. Distributed cooperative control system for smart microgrids. Electric Power Systems Research. 130, 241, 2016.

24. SHEN X., CAO M., ZHOU N., ZHANG L. Research on coordinated optimal scheduling and economic 
operation of multi-microgrid distribution system. JOURNAL OF ELECTRONIC MEASUREMENT AND INSTRUMENTATION. 30 (4), 568, 2016.

25. MALIK F.H., LEHTONEN M. A review:Agents in smart grids. Electric Power Systems Research. 131, 71, 2016.

26. LI X., YAO X. Cooperatively Coevolving Particle Swarms for Large Scale Optimization. IEEE TRANSACTIONS ON EVOLUTIONARY COMPUTATION. 16 (2), 210, 2012.

27. MAHDAVI S., SHIRI M.E., RAHNAMAYAN S. Metaheuristics in large-scale global continues optimization: A survey. Information Sciences. 295, 407, 2015.

28. YANG Z., TANG K., YAO X. Large scale evolutionary optimization using cooperative co-evolution. Information Sciences. 178, 2985, 2008.

29. YANG Z., TANG K., YAO X. Multilevel cooperative coevolution for large scale optimization. 2008 IEEE Congress on Evolutionary Computation (CEC). 1663, 2008.

30. OMIDVAR M., LI X., YAO X. Cooperative co-evolution with Delta grouping for large scale non-separable function optimization. 2010 IEEE Congress on Evolutionary Computation (CEC). 1762, 2010.

31. OMIDVAR M., LI X., MEI Y., YAO X. Cooperative co-evolution with differential grouping for large scale optimization. IEEE Transactions on Evolutionary Computation. 18 (3), 378, 2014.

32. SUN Y., KIRLEY M., HALGAMUGE S. K. Extended Differential Grouping for Large Scale Global Optimization with Direct and Indirect Variable Interactions. 2015 Genetic and Evolutionary Computation Conference (GECCO). 313, 2015.
33. YU Y., YU X., LI Y. Solving Engineering Optimization Problem by Augmented Lagrange Particle Swarm Optimization. JOURNAL OF MECHANICAL ENGINEERING. 45 (12), 167, 2009.

34. YANG Z., TANG K., YAO X. Self-adaptive differential evolution with neighborhood search. 2008 IEEE Congress on Evolutionary Computation (CEC). 1110, 2008.

35. WANG Y., CAI Z., ZHOU Y., FAN Z. Constrained optimization based on hybrid evolutionary algorithm and adaptive constraint-handling technique. Struct Multidisc Optim. 37, 395, 2009.

36. LIU D., GUO J., HUANG Y., WANG W. Dynamic Economic Dispatch of Wind Integrated Power System Based on Wind Power Probabilistic Forecasting and Operation Risk Constraints. Proceedings of the CESS. 33 (16), 9, 2013.

37. HONG T., PINSON P., FAN S., ZAREIPOUR H., TROCCOLI A., Hyndman R.J. Probabilistic energy forecasting: Global Energy Forecasting Competition 2014 and beyond. International Journal of Forecasting. 32, 896, 2016.

38. PRAGASPATHY, S., BASKARAN, A. Mitigation of Uncertainties in Wind-Powered Renewable Systems for Environmental Assets. Polish Journal of Environmental Studies. 26 (1), 253, 2017.

39. XIONG C., YANG D., HUO J., ZHAO Y. The Relationship between Agricultural Carbon Emissions and Agricultural Economic Growth and Policy Recommendations of a Low-carbon Agriculture Economy. Polish Journal of Environmental Studies. 25 (5), 2187, 2016. 